\title{
Approximate cloaking for the full wave equation via change of variables: The Drude-Lorentz model
}

\author{
Hoai-Minh Nguyen $^{\mathrm{a}, *}$, Michael S. Vogelius ${ }^{\mathrm{b}}$ \\ a EPFL SB MATHAA CAMA, Station 8, CH-1015 Lausanne, Switzerland \\ b Department of Mathematics, Rutgers University, New Brunswick, NJ 08903, USA
}

\section{A R T I C L E I N F O}

\section{Article history:}

Received 5 February 2016

Available online 21 March 2016

\section{$M S C$ : \\ 35B40 \\ $35 \mathrm{~L} 10$}

$74 \mathrm{E} 05$

Keywords:

Drude-Lorentz model

Wave equation

Approximate cloaking

Small inhomogeneity

\begin{abstract}
A B S T R A C T
This paper concerns approximate cloaking by mapping for a full, but scalar wave equation, when one allows for physically relevant frequency dependence of the material properties of the cloak. The paper is a natural continuation of [20], but here we employ the Drude-Lorentz model in the cloaking layer, that is otherwise constructed by an approximate blow up transformation of the type introduced in [10]. The central mathematical problem is to analyze the effect of a small inhomogeneity in the context of this non-local full wave equation.
\end{abstract}

() 2016 Elsevier Masson SAS. All rights reserved.

\section{R É S U M É}

L'article traite du « cloaking » approché par changement de variables pour l'équation des ondes scalaire avec amortissement. Il poursuit l'étude présentée dans [20] par l'étude d'un modèle réaliste et pertinent dans lesquels les coefficients constitutifs du milieu constituant la cape d'invisibilité dépendent de la fréquence. En l'occurence le dispositif est construit par une transformation asymptotiquement singulière analogue à celles introduites dans [10], cependant la région occupée par la cape d'invisibilité est décrite par un modèle de Drude-Lorentz. La question mathématique centrale de l'article est l'analyse de l'effet d'une inhomogénéité de petite taille dans le contexte de l'équation des ondes résultante, non locale avec amortissement.

() 2016 Elsevier Masson SAS. All rights reserved.

\section{Introduction}

Cloaking by mapping (frequently referred to as transformation optics) was introduced by Pendry, Schurig, and Smith [23] for the Maxwell system, and Leonhardt [12] in the geometric optics setting. These authors used a singular change of variables which blows up a point to a cloaked region. The exact same transformation

\footnotetext{
* Corresponding author.

E-mail addresses: hoai-minh.nguyen@epfl.ch (H.-M. Nguyen), vogelius@math.rutgers.edu (M.S. Vogelius).
} 
had been used before by Greenleaf, Lassas, and Uhlmann [6] to establish non-uniqueness in the context of the Calderon problem. The singular nature of the cloaks presents various difficulties in practice as well as in theory: (1) they are hard to fabricate and (2) in certain cases the correct definition of the corresponding electromagnetic fields is not obvious. To avoid the use of singular structures, regularized schemes have been proposed in $[3,4,10,26,27]$.

In this paper we analyze approximate cloaking for a full wave equation using transformation optics, where we incorporate the Drude-Lorentz model, see e.g., [8], in the layer constructed by transformation optics. The Drude-Lorentz model takes into account the effect of the oscillations of free electrons on the electric permittivity (by means of a simple harmonic oscillator model). We could have incorporated the same model in other parts of space, to better model conducting metallic elements of these parts as well. For the transformation optics construction we use the approximate scheme introduced in [10], which is based on a transformation blowing up a small ball of radius $\varepsilon$ to the cloaked region. When viewed in (complex) frequency domain, the refractive index associated with the Drude-Lorentz model may be extended analytically to the whole upper half plane. As is well known, an immediate consequence of this is causality for the associated non-local wave equation, see [8] and [28], - a property which is most essential for the well-posedness (and the physical relevance) of this equation. Another well known consequence of this analyticity property are the so-called Kramers-Krönig relations between the real and the imaginary part of the refractive index (they are essentially related by Hilbert transforms). However, this fact is not explicitly used in our analysis.

Approximate cloaking schemes for the Helmholtz equation based on the regularized transformations introduced in [10] have been studied extensively in various regimes, see [9,16,17,21]. A related scheme, which (in 3d) blows up a small diameter cylinder to the cloaked region was studied in [18] (see also [5,13, 14]). Frequently a (damping) lossy layer is employed inside the transformation cloak. Without this lossy layer, the field inside the cloaked region might depend on the field outside (even for a perfect cloak), and resonance can appear and destroy the cloaking (or approximate cloaking) ability of the pure transformation cloak, see [17].

We next describe the setting in detail. Given $r>0$, let $B_{r}$ denote the open ball centered at 0 and of radius $r$. Let $F_{\varepsilon}$ be the standard transformation $\mathbb{R}^{d} \rightarrow \mathbb{R}^{d}, d=2,3$, which blows up the ball $B_{\varepsilon}$ to $B_{1}$, equals the identity outside $B_{2}$, and is given by

$$
F_{\varepsilon}(x)=\left\{\begin{array}{cl}
x & \text { if } x \in \mathbb{R}^{d} \backslash B_{2}, \\
\left(\frac{2-2 \varepsilon}{2-\varepsilon}+\frac{|x|}{2-\varepsilon}\right) \frac{x}{|x|} & \text { if } x \in B_{2} \backslash B_{\varepsilon}, \\
\frac{x}{\varepsilon} & \text { if } x \in B_{\varepsilon} .
\end{array}\right.
$$

Assume that the cloaked region is the ball $B_{1 / 2}$, the contents of which is characterized by a real, matrix valued function $a$ and a complex function $\sigma$. The surrounding cloak contains two parts. In the time harmonic regime, these can be described as follows. The outer part is the Drude-Lorentz version of the standard layer, generated by the blow up map $F_{\varepsilon}$. In this layer, occupying $B_{2} \backslash B_{1}$, the material characteristics are given by

$$
\left(F_{\varepsilon}\right)_{*} I,\left(F_{\varepsilon}\right)_{*} 1+\sigma_{1, c}
$$

where

$$
\sigma_{1, c}(k, x)=\frac{\sigma_{N}}{k_{\varepsilon}^{2}-k^{2}-i \sigma_{D} k} .
$$

While the first part $\left(F_{\varepsilon}\right)_{*} 1$ of the refractive index in (1.2) is standard from the transformation optics approach, the second part $\sigma_{1, c}$ is exactly the correction introduced by the Drude-Lorentz model, see e.g., 
[8, page 331]. Here $\sigma_{N}$ and $\sigma_{D}$ are material constants which can in principle depend on the space variable $x$, and $k_{\varepsilon}>0$ is the so-called resonant frequency of the Drude-Lorentz model; in a more general model there could be several resonant frequencies $\left\{k_{i, \varepsilon}\right\}$, and the corresponding part of the refractive index would be a sum of terms (1.3) ranging over all these frequencies, see e.g., [8, page 310]. In this paper, we use the standard notation

$$
F_{*} A(y)=\frac{D F(x) A(x) D F^{T}(x)}{|\operatorname{det} D F(x)|}, \quad F_{*} \Sigma(y)=\frac{\Sigma(x)}{|\operatorname{det} D F(x)|}, \quad x=F^{-1}(y),
$$

for the "pushforward" of a symmetric, matrix valued function, $A$, and a scalar function, $\Sigma$, by the diffeomorphism $F$. In what follows, we assume for ease of notation that

$$
\sigma_{N}=\sigma_{D}=1 \text { in } B_{2} \backslash B_{1}
$$

The inner part of our cloak is a fixed damping layer as considered in [16]. This damping (lossy) layer occupies $B_{1} \backslash B_{1 / 2}$, and its material characteristics are given by

$$
I, 1+\frac{i}{k}
$$

Therefore, in the time harmonic regime, i.e., in frequency domain, the entire medium is characterized by ${ }^{1}$

$$
A_{c}, \Sigma_{c}:=\left\{\begin{array}{cc}
I, 1 & \text { in } \mathbb{R}^{d} \backslash B_{2}, \\
\left(F_{\varepsilon}\right)_{*} I,\left(F_{\varepsilon}\right)_{*} 1+\sigma_{1, c} & \text { in } B_{2} \backslash B_{1}, \\
I, 1+i / k & \text { in } B_{1} \backslash B_{1 / 2}, \\
a, \sigma & \text { in } B_{1 / 2} .
\end{array}\right.
$$

We assume that $a, \sigma \in L^{\infty}\left(B_{1 / 2}\right)$, with

$$
\frac{1}{\Lambda}|\xi|^{2} \leq\langle a \xi, \xi\rangle \leq \Lambda|\xi|^{2}, \quad \frac{1}{\Lambda} \leq \Re(\sigma) \leq \Lambda, \quad \text { and } \quad 0 \leq \Im(\sigma) \leq \Lambda
$$

for some positive constant $\Lambda$. With this notation, the temporal Fourier transform $\hat{u}_{c}$ of the field, ${ }^{2}$ will be a solution to

$$
\operatorname{div}\left(A_{c} \nabla \hat{u}_{c}\right)+k^{2} \Sigma_{c} \hat{u}_{c}=-\hat{f} .
$$

The temporal Fourier transform of a function $v(t, x)$ is given by

$$
\hat{v}(k, x)=\frac{1}{\sqrt{2 \pi}} \int_{-\infty}^{\infty} v(t, x) e^{i k t} d t
$$

The corresponding field in time domain (for positive time) is the unique weak solution $u_{c} \in L^{\infty}((0,+\infty)$; $\left.H^{1}\left(\mathbb{R}^{d}\right)\right)$, with $\partial_{t} u_{c} \in L^{\infty}\left([0,+\infty) ; L^{2}\left(\mathbb{R}^{d}\right)\right)$, to the non-local wave equation

\footnotetext{
1 Notice that the "damping layer", $B_{1} \backslash B_{1 / 2}$, is a bit different from that in [20] where, for any fixed $\gamma>0$, we used $A_{c}=I$, $\Sigma_{c}=$ $\varepsilon^{2}+\frac{i}{k \varepsilon^{\gamma}}$, for $n=2$, and $A_{c}=\varepsilon I, \Sigma_{c}=\varepsilon^{3}+\frac{i \varepsilon^{1-\gamma}}{k}$, for $n=3$. This change is, however, not essential - the essential change is in the layer $B_{2} \backslash B_{1}$, with the inclusion of $\sigma_{1, c}$. It would be interesting to investigate whether, in view of the damping present in $\sigma_{1, c}$, the layer $B_{1} \backslash B_{1 / 2}$ is necessary at all.

2 Where we extend the time domain field by 0 for negative time.
} 


$$
\left\{\begin{array}{cl}
\Sigma_{1, c} \partial_{t t}^{2} u_{c}-\operatorname{div}\left(A_{c} \nabla u_{c}\right)+\Sigma_{2, c} \partial_{t} u_{c}+G * \partial_{t} u_{c}=f & \text { in }[0,+\infty) \times \mathbb{R}^{d}, \\
\partial_{t} u_{c}(t=0)=u_{c}(t=0)=0 & \text { in } \mathbb{R}^{d},
\end{array}\right.
$$

where $f \in L^{2}\left((0,+\infty) \times \mathbb{R}^{d}\right)$ with compact support. The definition of weak solutions to (1.6), and the proof of well-posedness of (1.6) is presented in Section 4. The coefficients $\Sigma_{1, c}$ and $\Sigma_{2, c}$ are given by

$$
\Sigma_{1, c}=\left\{\begin{array}{cl}
1 & \text { in } \mathbb{R}^{d} \backslash B_{2}, \\
\left(F_{\varepsilon}\right)_{*} 1 & \text { in } B_{2} \backslash B_{1}, \\
1 & \text { in } B_{1} \backslash B_{1 / 2}, \\
\sigma & \text { in } B_{1 / 2},
\end{array} \quad \Sigma_{2, c}= \begin{cases}0 & \text { in } \mathbb{R}^{d} \backslash B_{2}, \\
0 & \text { in } B_{2} \backslash B_{1}, \\
1 & \text { in } B_{1} \backslash B_{1 / 2}, \\
0 & \text { in } B_{1 / 2},\end{cases}\right.
$$

and $G(t, x)$ is such that

$$
\widehat{G}(k, x)=-i k \sigma_{1, c}(k, x) \quad x \in B_{2} \backslash B_{1} .
$$

A computation (see, e.g., $[8,(7.110)])$ shows that

$$
G(t, x)=\phi(t) H(t)
$$

where $H(t)$ denotes the Heaviside function, i.e.,

$$
H(t)=\left\{\begin{array}{cc}
0 & \text { if } t<0 \\
1 & \text { otherwise }
\end{array}\right.
$$

and

$$
\phi(t)=\frac{\sqrt{2 \pi}}{\gamma_{0}} \partial_{t}\left(e^{-t / 2} \sin \left(\gamma_{0} t\right)\right)
$$

with

$$
\gamma_{0}=\sqrt{k_{\varepsilon}^{2}-1 / 4}
$$

We assume that $k_{\varepsilon}>1 / 2$, so that $\gamma_{0}$ is real and positive.

The presence of the Heaviside function in the formula (1.7) implies causality and plays an important role in our analysis; in particular for the proof of well-posedness of $u_{c}$, and to establish that the Fourier transform, $\hat{u}_{c}$, satisfies the outgoing radiation condition.

We only consider zero initial conditions. This is just for ease and simplicity of presentation; indeed, our method would work for the general case, using an approach similar to that in [20].

Given $f$, the corresponding field in the homogeneous medium without the cloak and the cloaked region is the unique weak solution $u \in L^{\infty}\left((0,+\infty) ; H^{1}\left(\mathbb{R}^{d}\right)\right)$, with $\partial_{t} u \in L^{\infty}\left((0,+\infty) ; L^{2}\left(\mathbb{R}^{d}\right)\right)$, to the system

$$
\left\{\begin{array}{cl}
\partial_{t t}^{2} u-\Delta u=f & \text { in }(0,+\infty) \times \mathbb{R}^{d}, \\
\partial_{t} u(t=0)=u(t=0)=0 & \text { in } \mathbb{R}^{d} .
\end{array}\right.
$$

The extent to which we have succeeded in hiding the contents of $B_{1 / 2}$ and the cloak itself, should be measured in terms of the difference between $u_{c}$ and $u$, outside $B_{2}$. The main Theorem of this paper gives an estimate of this difference for the scheme in (1.4). 
Theorem 1. Let $d=2$ or 3 , and let $f \in C^{\infty}\left([0,+\infty) \times \mathbb{R}^{d}\right)$ be such that $\operatorname{supp} f \subset(0, R) \times\left(B_{R} \backslash B_{2}\right)$ for some $R>0$. Suppose $c_{*} \varepsilon^{-d / 2}<k_{\varepsilon}<C_{*} \varepsilon^{-K}$ for some positive constant $c_{*}, C_{*}$ and $K>d / 2$. Given any integer $M \geq 2 d+4 K-2$, there exists a constant $C$ such that

$$
\sup _{0<t<T}\left\|u_{c}-u\right\|_{L^{2}\left(B_{5} \backslash B_{2}\right)} \leq C \varepsilon T\|f\|_{C^{M}\left([0, R] ; L^{2}\left(B_{R}\right)\right)} \quad \forall T>0, \quad \text { for } d=3
$$

and

$$
\sup _{0<t<T}\left\|u_{c}-u\right\|_{L^{2}\left(B_{5} \backslash B_{2}\right)} \leq C \frac{1}{|\ln \varepsilon|} T\|f\|_{C^{M}\left([0, R] ; L^{2}\left(B_{R}\right)\right)} \quad \forall T>0, \quad \text { for } d=2 .
$$

$C$ depends on $R, c_{*}, C_{*}, K$ and $M$, but is independent of $f, \varepsilon, k_{\varepsilon}, \Lambda$ and $T$.

We in fact prove the following slightly stronger result:

Theorem 2. Let $d=2$ or 3 , and let $f \in C^{\infty}\left([0,+\infty) \times \mathbb{R}^{d}\right)$ be such that $\operatorname{supp} f \subset(0, R) \times\left(B_{R} \backslash B_{2}\right)$ for some $R>0$. Suppose $k_{\varepsilon}>c_{*} \varepsilon^{-d / 2}$ for some positive constant $c_{*}$, then

$$
\sup _{0<t<T}\left\|u_{c}-u\right\|_{L^{2}\left(B_{5} \backslash B_{2}\right)} \leq C \varepsilon T\|f\| \quad \forall T>0, \quad \text { for } d=3
$$

and

$$
\sup _{0<t<T}\left\|u_{c}-u\right\|_{L^{2}\left(B_{5} \backslash B_{2}\right)} \leq C \frac{1}{|\ln \varepsilon|} T\|f\| \quad \forall T>0, \quad \text { for } d=2 .
$$

Here $C$ is a positive constant depending on $R$ and $c_{*}$, but independent of $f, \varepsilon, k_{\varepsilon}, \Lambda$ and $T$. The norm of $f$ is defined by

$$
\|f\|=\|f\|_{k_{\varepsilon}, \varepsilon}:=\int_{0}^{\infty}\left(1+k^{2 d+1}\right)\|\hat{f}(k, \cdot)\|_{L^{2}} d k+\int_{\lambda_{0} / \varepsilon}^{\infty} k^{2 d-3} k_{\varepsilon}^{4}\|\hat{f}(k, \cdot)\|_{L^{2}} d k
$$

for some fixed positive constant $\lambda_{0}$, depending only on $c_{*}$. Here $\hat{f}$ is the Fourier transform of $f$ with respect to time, $f$ being extended by zero for $t<0$.

The assumption that $\operatorname{supp} f \subset(0, R) \times\left(B_{R} \backslash B_{2}\right)$ could be replaced by $\operatorname{supp} f \subset[0, R) \times\left(B_{R} \backslash B_{2}\right)$ (i.e., $f$ does not have to vanish in a neighborhood of $t=0)$ provided one assumes that $\tilde{f} \in C^{\infty}\left((-\infty,+\infty) \times \mathbb{R}^{d}\right)$, where $\tilde{f}$ denotes the extension of $f$ by zero for $t<0$. The condition that $f$ or $\tilde{f}$ be in $C^{\infty}$ could also be replaced by an assumption about the continuity of only finitely many derivatives. We leave the details to the reader.

Theorem 1 follows directly from Theorem 2 by noting that if $c_{*} \varepsilon^{-d / 2}<k_{\varepsilon}<C_{*} \varepsilon^{-K}$, for some $K>d / 2$, then

$$
\begin{aligned}
\|f\| & =\int_{0}^{\infty}\left(1+k^{2 d+1}\right)\|\hat{f}(k, \cdot)\|_{L^{2}}+\int_{\lambda_{0} / \varepsilon}^{\infty} k^{2 d-3} k_{\varepsilon}^{4}\|\hat{f}(k, \cdot)\|_{L^{2}} \\
& \leq C \int_{0}^{\infty}\left(1+k^{2 d+4 K-3}\right)\|\hat{f}(k, \cdot)\|_{L^{2}}
\end{aligned}
$$




$$
\begin{aligned}
& \leq C\left(\int_{0}^{\infty}(1+k)^{-2}\right)^{1 / 2}\left(\int_{0}^{\infty}\left(1+k^{2 d+4 K-2}\right)^{2}\|\hat{f}(k, \cdot)\|_{L^{2}}^{2}\right)^{1 / 2} \\
& \leq C\|f\|_{C^{M}\left([0, R] ; L^{2}\left(B_{R}\right)\right)}
\end{aligned}
$$

for any integer $M \geq 2 d+4 K-2$. Here we used that supp $f \subset(0, R) \times\left(B_{R} \backslash B_{2}\right)$ so that the $C^{M}$-norm of the extension of $f$ by zero for $t<0$ is bounded by $\|f\|_{C^{M}\left([0, R] ; L^{2}\left(B_{R}\right)\right)}$.

The results obtained in this paper are in a slightly different spirit than the ones in [20] (and, of course, for a different problem). The constants in Theorem 2 and Theorem 1 here are independent of $\Lambda$, while the ones in [20, Theorems 1 and 2] are not. However, the estimates in [20, Theorems 1 and 2] are uniform in time, while the ones in Theorem 2 and Theorem 1 here are not. The independence of the constants of $\Lambda$ yields a stronger result about the cloaking effects, since it asserts that the cloak works well for arbitrary objects. Similar results as in [20] (i.e., results that are uniform in time, but not in $\Lambda$ ) would hold in this setting, and results of the type in Theorem 2 and Theorem 1 would hold in the setting of [20].

The approach in this paper borrows several ideas from the approach in [20], and adapts these to the setting considered here. We transform the wave equation into a family of Helmholtz equations by taking the Fourier transform with respect to time. Having established the appropriate near-invisibility estimates for the Helmholtz equations, with explicit frequency dependence, we then essentially invert the Fourier transform. As concerns the Helmholtz equations, we study and compare the model with $\sigma_{1, c}$ in (1.4) and the model without $\sigma_{1, c}$, and establish perturbation estimates in the time harmonic regime. Note that, for the model with $\sigma_{1, c}$, the standard rescaling techniques, as used in [16,17,20,21], do not work. We hence work directly with this model without rescaling (Section 3). The proof is quite delicate, makes use of many ideas from $[16,17,20,21]$, and at a crucial point requires an argument of "removable singularity" (in the proof of Lemma 5). To obtain the estimates in time domain from the estimates in frequency domain, we proceed in a similar, but slightly different way than [20]. We use a simple and helpful idea, also used in [19], by establishing estimates for the difference of the time derivatives of $u_{c}$ and $u$ not for their difference. As a consequence, we avoid the non-standard estimates for very low frequency in [20, Section 2.2]; their proof involved the theory of $H$-convergence. Moreover, using this idea, we are also able to obtain the independence of $\Lambda$ for the constants in Theorem 2. As mentioned earlier, another element of our analysis is the (definition of and) verification of well-posedness of $u_{c}$ (Proposition 1). For this purpose we rely on a non-trivial energy estimate, in the spirit of [19].

The paper is organized as follows. In Section 2, we present results for the model without $\sigma_{1, c}$ and some estimates for $\left(F_{\varepsilon}^{-1}\right)_{*} \sigma_{1, c}$. These will be used in the proof of Theorem 2 to obtain estimates in the time harmonic regime, when the frequency is of order at most $1 / \varepsilon$. Section 3 provides estimates for $u_{c}$ in the time harmonic regime for arbitrarily large frequencies. In section 4, we establish the well-posedness of $u_{c}$ and discuss the outgoing radiation condition for its Fourier transform with respect to time. The required non-trivial energy estimate for $u_{c}$ is also derived there. Finally, the proof of Theorem 2 is given in Section 5.

\section{Preliminaries}

In this section we recall some known results, which will be used frequently in this paper, and we derive an estimate related to the model without $\sigma_{1, c}$ in the time harmonic regime, when the frequency is of order at most $1 / \varepsilon$. This estimate is an extension of $\left[16\right.$, Lemma 2.4]. We also estimate $\left(F_{\varepsilon}^{-1}\right)_{*} \sigma_{1, c}$ in various regions. These results will be used in Section 5 in the proof of Theorem 2.

Let $U$ denote a connected smooth open region of $\mathbb{R}^{d}(d=2$ or 3$)$ with a bounded complement (this includes $\left.U=\mathbb{R}^{d}\right)$. Here and in what follows, a solution $v \in H_{l o c}^{1}(U)(d=2$ or 3$)$ to the Helmholtz equation

$$
\Delta v+k^{2} v=0 \text { in } U
$$


for some $k>0$, is said to be an outgoing solution (or satisfy the outgoing radiation condition) if

$$
\frac{\partial v}{\partial r}-i k v=o\left(r^{-\frac{d-1}{2}}\right) \text { as } r \rightarrow \infty .
$$

We shall also need the space $W^{1}(U)$; it is defined as follows,

$$
W^{1}(U)=\left\{\psi \in L_{l o c}^{1}(U): \frac{\psi(x)}{\sqrt{1+|x|^{2}}} \in L^{2}(U) \text { and } \nabla \psi \in L^{2}(U)\right\} \quad \text { for } d=3,
$$

and,

$$
W^{1}(U)=\left\{\psi \in L_{l o c}^{1}(U): \frac{\psi(x)}{\ln (2+|x|) \sqrt{1+|x|^{2}}} \in L^{2}(U) \text { and } \nabla \psi \in L^{2}(U)\right\} \quad \text { for } d=2 .
$$

Lemma 1. Let $d=2$ or 3 and $k>0$. Suppose $f \in L^{2}\left(\mathbb{R}^{d}\right)$ with $\operatorname{supp} f \subset B_{5}$, and let $v_{k} \in H_{\text {loc }}^{1}\left(\mathbb{R}^{d}\right)$ be the unique outgoing solution to

$$
\Delta v_{k}+k^{2} v_{k}=f \text { in } \mathbb{R}^{d}
$$

Then, for $d=2$ and $0 \leq k \leq 1 / 2$,

$$
\left\|\nabla v_{k}\right\|_{L^{2}\left(B_{6}\right)}+\left\|v_{k}\right\|_{L^{2}\left(B_{6}\right)} \leq C|\ln k|\|f\|_{L^{2}},
$$

and for $d=3$ or for $d=2$ and $k>1 / 2$,

$$
\left\|\nabla v_{k}\right\|_{L^{2}\left(B_{6}\right)}+(k+1)\left\|v_{k}\right\|_{L^{2}\left(B_{6}\right)} \leq C\|f\|_{L^{2}} .
$$

Here $C$ is a positive constant independent of $k$ and $f$.

Proof. The conclusion in the case $k<k_{0}$, for arbitrary fixed $k_{0}>0$, follows directly from the properties of the fundamental solution to the Helmholtz equation. The conclusion in the case $k \geq k_{0}$ can also be obtained from the fundamental solution to the Helmholtz equation. In this case, one can alternately obtain the conclusion using the Morawetz multipliers (see, e.g., [21, Lemma 2 and Proposition 1]). We note that the estimate in [21, Proposition 1] requires a damping layer due to the desire to obtain estimates that are independent of the arbitrary coefficients inside $B_{1 / 2}$. Since the operator here is $\Delta+k^{2}$ throughout, there is no need for such a layer. The details are left to the reader.

We next recall the following result which will be used frequently in this paper. The result is from [16, Lemma 2.2] (see also [21, Lemma 3]).

Lemma 2. Let $d=2$ or 3 , and let $D$ be a smooth, open bounded subset of $\mathbb{R}^{d}$ such that $\mathbb{R}^{d} \backslash D$ is connected. Suppose $0<k<\tau$, for some fixed $\tau>0$, and suppose $g_{k} \in H^{1 / 2}(\partial D)$. Let $v_{k} \in H_{\text {loc }}^{1}\left(\mathbb{R}^{d} \backslash D\right)$ be the unique outgoing solution to

$$
\left\{\begin{array}{cl}
\Delta v_{k}+k^{2} v_{k}=0 & \text { in } \mathbb{R}^{d} \backslash D \\
v_{k}=g_{k} & \text { on } \partial D
\end{array}\right.
$$

Then

$$
\left\|v_{k}\right\|_{H^{1}\left(B_{R} \backslash D\right)} \leq C_{R}\left\|g_{k}\right\|_{H^{1 / 2}(\partial D)} \quad \text { for any } R>0
$$


The constant $C_{R}$ is independent of $k$ and $g_{k}$. Furthermore for any $\varepsilon>0$ sufficiently small that $D \subset B_{2 / \varepsilon}$

$$
\left\{\begin{array}{c}
\left\|v_{k}\right\|_{L^{2}\left(B_{5 / \varepsilon} \backslash B_{2 / \varepsilon}\right)} \leq C \varepsilon^{-1 / 2}\left\|g_{k}\right\|_{H^{1 / 2}(\partial D)} \quad \text { if } \quad d=3 \\
\left\|v_{k}\right\|_{L^{2}\left(B_{5 / \varepsilon} \backslash B_{2 / \varepsilon}\right)} \leq C \varepsilon^{-1} \frac{\left|H_{0}^{(1)}(k / \varepsilon)\right|}{\left|H_{0}^{(1)}(k)\right|}\left\|g_{k}\right\|_{H^{1 / 2}(\partial D)} \quad \text { if } \quad d=2 .
\end{array}\right.
$$

Here the constant $C$ is independent of $k, g_{k}$ and $\epsilon$. Finally, if we assume that $g_{k} \rightarrow g$ weakly in $H^{1 / 2}(\partial D)$ as $k \rightarrow 0$, then $v_{k} \rightarrow v$ weakly in $H_{\text {loc }}^{1}\left(\mathbb{R}^{d} \backslash D\right)$ where $v \in W^{1}\left(\mathbb{R}^{d} \backslash D\right)$ is the unique solution of

$$
\left\{\begin{array}{cl}
\Delta v=0 & \text { in } \mathbb{R}^{d} \backslash D, \\
v=g & \text { on } \partial D .
\end{array}\right.
$$

We next establish an estimate for the model without $\sigma_{1, c}$, for frequency at most $1 / \varepsilon$.

Lemma 3. Let $d=2$ or 3 , and let $a$ and $\sigma$ be in $L^{\infty}\left(B_{1 / 2}\right)$, with

$$
\text { a real symmetric, uniformly positive definite, and } \Im(\sigma) \geq 0 \text {. }
$$

Suppose $0<\varepsilon<\tau$, and $0<k<\tau / \varepsilon$ for some fixed, positive constant $\tau$. For $g \in H^{-\frac{1}{2}}\left(\partial B_{1}\right)$ let $v_{\varepsilon} \in H_{\text {loc }}^{1}\left(\mathbb{R}^{d}\right)$ be the unique outgoing solution to

$$
\begin{cases}\Delta v_{\varepsilon}+\varepsilon^{2} k^{2} v_{\varepsilon}=0 & \text { in } \mathbb{R}^{d} \backslash \bar{B}_{1}, \\ \operatorname{div}\left(A \nabla v_{\varepsilon}\right)+k^{2} \Sigma v_{\varepsilon}=0 & \text { in } B_{1}, \\ \left.\frac{\partial v_{\varepsilon}}{\partial r}\right|_{\text {ext }}-\left.\frac{1}{\varepsilon^{d-2}} \frac{\partial v_{\varepsilon}}{\partial r}\right|_{\text {int }}=g & \text { on } \partial B_{1} .\end{cases}
$$

Here

$$
A=\left\{\begin{array}{ll}
I \quad \text { if } x \in \mathbb{R}^{d} \backslash B_{1 / 2}, \\
a \quad \text { if } x \in B_{1 / 2},
\end{array} \quad \Sigma=\left\{\begin{array}{cl}
1 & \text { if } x \in \mathbb{R}^{d} \backslash B_{1}, \\
1+i / k & \text { if } x \in B_{1} \backslash B_{1 / 2}, \\
\sigma & \text { if } x \in B_{1 / 2} .
\end{array}\right.\right.
$$

There exists a positive constant $C$, depending only on $d$ and $\tau$, such that

$$
\left\|v_{\varepsilon}\right\|_{H^{1}\left(B_{5} \backslash B_{1}\right)} \leq C \max \left\{k^{3-d}, \varepsilon^{d-2} / k\right\}\|g\|_{H^{-\frac{1}{2}}\left(\partial B_{1}\right)} .
$$

Proof of Lemma 3. We follow the strategy in the proof of [16, Lemma 2.4], and consider the case $d=2$ and $d=3$ separately.

Case 1: $d=2$. We first prove

$$
\left\|v_{\varepsilon}\right\|_{L^{2}\left(B_{5} \backslash B_{1}\right)} \leq C \max \{k, 1 / k\}\|g\|_{H^{-\frac{1}{2}}\left(\partial B_{1}\right)},
$$

by contradiction. Suppose this estimate is not true. Then there exist $\left(g_{n}\right) \subset H^{-\frac{1}{2}}\left(\partial B_{1}\right),\left(\varepsilon_{n}\right),\left(k_{n}\right),\left(a_{n}\right)$, and $\left(\sigma_{n}\right)$ such that $0<\varepsilon_{n}<\tau, 0<k_{n}<\tau / \varepsilon_{n}, a_{n}$ and $\sigma_{n}$ satisfy (2.1), and

$$
\left\|v_{n}\right\|_{L^{2}\left(B_{5} \backslash B_{1}\right)}=1 \quad, \quad \lim _{n \rightarrow \infty} \max \left\{k_{n}, 1 / k_{n}\right\}\left\|g_{n}\right\|_{H^{-\frac{1}{2}}\left(\partial B_{1}\right)}=0 .
$$


Here $v_{n} \in H_{l o c}^{1}\left(\mathbb{R}^{2}\right)$ is the unique outgoing solution to

$$
\begin{cases}\Delta v_{n}+\varepsilon_{n}^{2} k_{n}^{2} v_{n}=0 & \text { in } \mathbb{R}^{2} \backslash B_{1}, \\ \operatorname{div}\left(A_{n} \nabla v_{n}\right)+k_{n}^{2} \Sigma_{n} v_{n}=0 & \text { in } B_{1}, \\ \left.\frac{\partial v_{n}}{\partial r}\right|_{\text {ext }}-\left.\frac{\partial v_{n}}{\partial r}\right|_{\text {int }}=g_{n} & \text { on } \partial B_{1}\end{cases}
$$

where $A_{n}$ and $\Sigma_{n}$ are defined the same way as $A$ and $\Sigma$, with $a$ and $\sigma$ replaced by $a_{n}$ and $\sigma_{n}$. Multiplying the equation for $v_{n}$ by $\bar{v}_{n}$ (the conjugate of $v_{n}$ ) and integrating on $B_{R}$, we obtain

$$
\begin{gathered}
\int_{\partial B_{R}} \partial_{r} v_{n} \bar{v}_{n}-\int_{B_{R} \backslash B_{1}}\left|\nabla v_{n}\right|^{2}+\varepsilon_{n}^{2} k_{n}^{2} \int_{B_{R} \backslash B_{1}}\left|v_{n}\right|^{2} \\
-\int_{B_{1}}\left\langle A_{n} \nabla v_{n}, \nabla \bar{v}_{n}\right\rangle+k_{n}^{2} \int_{B_{1}} \Sigma_{n}\left|v_{n}\right|^{2}=\int_{\partial B_{1}} g_{n} \bar{v}_{n} .
\end{gathered}
$$

Letting $R \rightarrow \infty$ in (2.6), using the outgoing condition, and considering the imaginary part, we derive that

$$
k_{n} \int_{B_{1} \backslash B_{1 / 2}}\left|v_{n}\right|^{2} \leq\left\|g_{n}\right\|_{H^{-1 / 2}\left(\partial B_{1}\right)}\left\|v_{n}\right\|_{H^{1 / 2}\left(\partial B_{1}\right)} .
$$

By Caccioppoli's inequality, it follows that

$$
\begin{aligned}
\int_{B_{4 / 5} \backslash B_{3 / 5}}\left|\nabla v_{n}\right|^{2} & \leq C\left(k_{n}^{2}+1\right) \int_{B_{1} \backslash B_{1 / 2}}\left|v_{n}\right|^{2} \\
& \leq C \max \left\{k_{n}, 1 / k_{n}\right\}\left\|g_{n}\right\|_{H^{-1 / 2}\left(\partial B_{1}\right)}\left\|v_{n}\right\|_{H^{1 / 2}\left(\partial B_{1}\right)} .
\end{aligned}
$$

Here and in the remainder of this proof, $C$ denotes a positive constant depending only on $d$ and $\tau$ (which might change from one place to another). The above estimate implies that for some $r \in(3 / 5,4 / 5)(r$ depends on $n)$,

$$
\int_{\partial B_{r}}\left|\nabla v_{n}\right|^{2}+\left(1+k_{n}^{2}\right) \int_{\partial B_{r}}\left|v_{n}\right|^{2} \leq C \max \left\{k_{n}, 1 / k_{n}\right\}\left\|g_{n}\right\|_{H^{-1 / 2}\left(\partial B_{1}\right)}\left\|v_{n}\right\|_{H^{1 / 2}\left(\partial B_{1}\right)} .
$$

Multiplying the equation for $v_{n}$ by $\bar{v}_{n}$ and integrating on $B_{5} \backslash B_{r}$, we have

$$
\begin{aligned}
& \int_{\partial B_{5}} \partial_{r} v_{n} \bar{v}_{n}-\int_{\partial B_{r}} \partial_{r} v_{n} \bar{v}_{n}-\int_{B_{5} \backslash B_{r}}\left|\nabla v_{n}\right|^{2}+\varepsilon_{n}^{2} k_{n}^{2} \int_{B_{5} \backslash B_{1}}\left|v_{n}\right|^{2} \\
& +k_{n}^{2} \int_{B_{1} \backslash B_{r}} \Sigma_{n}\left|v_{n}\right|^{2}=\int_{\partial B_{1}} g_{n} \bar{v}_{n} .
\end{aligned}
$$

Since $v_{n} \in H_{l o c}^{1}\left(\mathbb{R}^{2} \backslash B_{3}\right)$ is the unique outgoing solution to $\Delta v_{n}+\varepsilon_{n}^{2} k_{n}^{2} v_{n}=0$ in $\mathbb{R}^{2} \backslash B_{3}$ and $\varepsilon_{n} k_{n} \leq \tau$, it follows that (see, e.g., Lemma 2)

$$
\left\|v_{n}\right\|_{H^{1}\left(B_{6} \backslash B_{3}\right)} \leq C\left\|v_{n}\right\|_{H^{1 / 2}\left(\partial B_{3}\right)} .
$$

Since $\Delta v_{n}+\varepsilon_{n}^{2} k_{n}^{2} v_{n}=0$ in $B_{5} \backslash B_{1}$, using the standard theory of elliptic equations, we have that 


$$
\left\|v_{n}\right\|_{H^{1 / 2}\left(\partial B_{3}\right)} \leq C\left\|v_{n}\right\|_{H^{1}\left(B_{4} \backslash B_{2}\right)} \leq C\left\|v_{n}\right\|_{L^{2}\left(B_{5} \backslash B_{1}\right)} .
$$

A combination of (4.25) and (4.26) yields

$$
\left\|v_{n}\right\|_{H^{1}\left(B_{6} \backslash B_{3}\right)} \leq C\left\|v_{n}\right\|_{L^{2}\left(B_{5} \backslash B_{1}\right)} \cdot
$$

Using (2.7), (2.8), and (2.12), we derive from (2.9) that

$$
\int_{B_{5} \backslash B_{r}}\left|\nabla v_{n}\right|^{2} \leq C \max \left\{k_{n}, 1 / k_{n}\right\}\left\|g_{n}\right\|_{H^{-1 / 2}\left(\partial B_{1}\right)}\left\|v_{n}\right\|_{H^{1 / 2}\left(\partial B_{1}\right)}+C\left\|v_{n}\right\|_{L^{2}\left(B_{5} \backslash B_{1}\right)}^{2} .
$$

We immediately obtain from (2.13) that

$$
\begin{aligned}
& \int_{B_{5} \backslash B_{1}}\left|\nabla v_{n}\right|^{2}+\int_{B_{5} \backslash B_{1}}\left|v_{n}\right|^{2} \\
& \quad \leq C \max \left\{k_{n}, 1 / k_{n}\right\}\left\|g_{n}\right\|_{H^{-1 / 2}\left(\partial B_{1}\right)}\left\|v_{n}\right\|_{H^{1 / 2}\left(\partial B_{1}\right)}+C\left\|v_{n}\right\|_{L^{2}\left(B_{5} \backslash B_{1}\right)}^{2} \\
& \leq C\left(\max \left\{k_{n}, 1 / k_{n}\right\}\left\|g_{n}\right\|_{H^{-1 / 2}\left(\partial B_{1}\right)}+\left\|v_{n}\right\|_{L^{2}\left(B_{5} \backslash B_{1}\right)}\right)\left\|v_{n}\right\|_{H^{1}\left(B_{5} \backslash B_{1}\right)}
\end{aligned}
$$

and so, by (2.4)

$$
\left\|v_{n}\right\|_{H^{1}\left(B_{5} \backslash B_{1}\right)} \leq C, \quad \text { and } \quad\left\|v_{n}\right\|_{H^{1 / 2}\left(\partial B_{1}\right)} \leq C .
$$

From (2.7) and (2.15), we conclude

$$
\left(1+k_{n}^{2}\right) \int_{B_{1} \backslash B_{1 / 2}}\left|v_{n}\right|^{2} \leq C \max \left\{k_{n}, 1 / k_{n}\right\}\left\|g_{n}\right\|_{H^{-1 / 2}\left(\partial B_{1}\right)},
$$

so by $(2.4)$

$$
\lim _{n \rightarrow \infty}\left(1+k_{n}^{2}\right) \int_{B_{1} \backslash B_{1 / 2}}\left|v_{n}\right|^{2}=0
$$

Since, for any $v \in H^{1}\left(B_{1} \backslash B_{r}\right)$,

$$
\|v\|_{L^{2}\left(\partial B_{1}\right)}^{2} \leq C\|v\|_{L^{2}\left(B_{1} \backslash B_{r}\right)}\|v\|_{H^{1}\left(B_{1} \backslash B_{r}\right)},
$$

(see [7, Lemma 5.5]), it follows from (2.13), (2.15), and (2.16) that

$$
\lim _{n \rightarrow \infty}\left\|v_{n}\right\|_{L^{2}\left(\partial B_{1}\right)}=0
$$

We have (see, e.g., Lemma 2) for any $R>1$,

$$
\left\|v_{n}\right\|_{H^{1}\left(B_{R} \backslash B_{1}\right)} \leq C_{R}\left\|v_{n}\right\|_{H^{1 / 2}\left(\partial B_{1}\right)} \leq C_{R},
$$

where we used the second estimate of (2.15) to obtain the last bound. By extraction of a subsequence (and a diagonalization argument) one might assume that $\varepsilon_{n} k_{n} \rightarrow \omega \in[0, \tau]$ (since $\varepsilon_{n} k_{n} \in[0, \tau]$ ) and $v_{n} \rightarrow v$ weakly in $H_{l o c}^{1}\left(\mathbb{R}^{2} \backslash B_{1}\right),\left.v_{n}\right|_{\partial B_{1}} \rightarrow 0$ weakly in $H^{1 / 2}\left(\partial B_{1}\right)$. By $(2.4)$, 


$$
\|v\|_{L^{2}\left(B_{5} \backslash B_{1}\right)}=1
$$

and for $\omega>0, v$ is the unique outgoing solution $t^{3}$

$$
\left\{\begin{array}{cl}
\Delta v+\omega^{2} v=0 & \text { in } \mathbb{R}^{2} \backslash B_{1} \\
v=0 & \text { on } \partial B_{1}
\end{array}\right.
$$

Hence $v=0$, and so we have a contradiction to (2.17). If $\omega=0$, then by Lemma $2, v \in W^{1}\left(\mathbb{R}^{2}\right)$ is the unique such solution to

$$
\left\{\begin{array}{cl}
\Delta v=0 & \text { in } \mathbb{R}^{2} \backslash B_{1} \\
v=0 & \text { on } \partial B_{1}
\end{array}\right.
$$

Hence $v=0$, and so again we have a contradiction to (2.17). This verifies the $L^{2}$ estimate (2.3). We have, as in (2.13),

$$
\int_{B_{5} \backslash B_{1}}\left|\nabla v_{\varepsilon}\right|^{2} \leq C \max \{k, 1 / k\}\|g\|_{H^{-1 / 2}\left(\partial B_{1}\right)}\left\|v_{\varepsilon}\right\|_{H^{1 / 2}\left(\partial B_{1}\right)}+C \int_{B_{5} \backslash B_{1}}\left|v_{\varepsilon}\right|^{2},
$$

and so by $(2.3)$

$$
\left\|v_{\varepsilon}\right\|_{H^{1}\left(B_{5} \backslash B_{1}\right)} \leq C \max \{k, 1 / k\}\|g\|_{H^{-1 / 2}\left(\partial B_{1}\right)}
$$

as desired. This completes the proof of the lemma for $d=2$.

$\underline{\text { Case 2: }} d=3$. We have (see, e.g., Lemma 2)

$$
\left\|v_{\varepsilon}\right\|_{H^{1}\left(B_{5} \backslash B_{1}\right)} \leq C\left\|v_{\varepsilon}\right\|_{H^{1 / 2}\left(\partial B_{1}\right)} .
$$

Hence it suffices to prove that

$$
\left\|v_{\varepsilon}\right\|_{H^{\frac{1}{2}}\left(\partial B_{1}\right)} \leq C \max \{1, \varepsilon / k\}\|g\|_{H^{-\frac{1}{2}}\left(\partial B_{1}\right)} .
$$

We first prove (2.19) by contradiction for $\varepsilon \leq \varepsilon_{0}$, with $\varepsilon_{0}$ sufficiently small. Suppose this is not true. Then there exist $\left(g_{n}\right) \subset H^{-\frac{1}{2}}\left(\partial B_{1}\right),\left(\varepsilon_{n}\right),\left(k_{n}\right),\left(a_{n}\right)$, and $\left(\sigma_{n}\right)$ such that $0<\varepsilon_{n}<\tau, 0<k_{n}<\tau / \varepsilon_{n}, a_{n}$ and $\sigma_{n}$ satisfy (2.1), $\varepsilon_{n} \rightarrow 0$, and

$$
\left\|v_{n}\right\|_{H^{\frac{1}{2}}\left(\partial B_{1}\right)}=1, \quad \lim _{n \rightarrow \infty} \max \left\{1, \varepsilon_{n} / k_{n}\right\}\left\|g_{n}\right\|_{H^{-\frac{1}{2}}\left(\partial B_{1}\right)}=0
$$

Here $v_{n} \in H_{l o c}^{1}\left(\mathbb{R}^{3}\right)$ is the unique outgoing solution to

$$
\begin{cases}\Delta v_{n}+\varepsilon_{n}^{2} k_{n}^{2} v_{n}=0 & \text { in } \mathbb{R}^{3} \backslash B_{1} \\ \operatorname{div}\left(A_{n} \nabla v_{n}\right)+k_{n}^{2} \Sigma_{n} v_{n}=0 & \text { in } B_{1} \\ \left.\frac{\partial v_{n}}{\partial r}\right|_{\text {ext }}-\left.\frac{1}{\varepsilon_{n}} \frac{\partial v_{n}}{\partial r}\right|_{\text {int }}=g_{n} & \text { on } \partial B_{1}\end{cases}
$$

\footnotetext{
3 The outgoing property of $v$ is just a consequence of the fact that the fundamental solution of the Helmholz equation with frequency $\varepsilon_{n} k_{n}$ converges to the fundamental solution of the Helmholtz equation with frequency $\omega$, since $\omega>0$.
} 
where $A_{n}$ and $\Sigma_{n}$ are defined in the same way as $A$ and $\Sigma$, but with $a$ and $\sigma$ replaced by $a_{n}$ and $\sigma_{n}$. Since $\left\|v_{n}\right\|_{H^{\frac{1}{2}}\left(\partial B_{1}\right)}=1$, it follows from (2.18) that

$$
\left\|v_{n}\right\|_{H^{1}\left(B_{5} \backslash B_{1}\right)} \leq C
$$

In combination with (2.20), (2.21), and the fact that $\varepsilon_{n} \rightarrow 0$ this implies

$$
\lim _{n \rightarrow \infty}\left\|\left.\frac{\partial v_{n}}{\partial r}\right|_{\text {int }}\right\|_{H^{-\frac{1}{2}\left(\partial B_{1}\right)}}=0
$$

Multiplying the equation of $v_{n}$ by $\bar{v}_{n}$ and integrating on $B_{R}$, we obtain

$$
\begin{aligned}
& \int_{\partial B_{R}} \partial_{r} v_{n} \bar{v}_{n}-\int_{B_{R} \backslash B_{1}}\left|\nabla v_{n}\right|^{2}+\varepsilon_{n}^{2} k_{n}^{2} \int_{B_{R} \backslash B_{1}}\left|v_{n}\right|^{2} \\
& \quad-\frac{1}{\varepsilon_{n}} \int_{B_{1}}\left\langle A_{n} \nabla v_{n}, \nabla \bar{v}_{n}\right\rangle+\frac{k_{n}^{2}}{\varepsilon_{n}} \int_{B_{1}} \Sigma_{n}\left|v_{n}\right|^{2}=\int_{\partial B_{1}} g_{n} \bar{v}_{n} .
\end{aligned}
$$

Letting $R \rightarrow \infty$ in (2.23), using the outgoing condition, and considering the imaginary part, we derive from (2.20) and the fact $k_{n} \varepsilon_{n} \leq \tau$ that

$$
\lim _{n \rightarrow+\infty}\left(1+k_{n}^{2}\right) \int_{B_{1} \backslash B_{1 / 2}}\left|v_{n}\right|^{2}=0
$$

Since $\Delta v_{n}+k_{n}^{2}\left(1+i / k_{n}\right) v_{n}=0$ in $B_{1} \backslash B_{1 / 2}$, by Caccioppoli's inequality, we obtain

$$
\int_{B_{4 / 5} \backslash B_{3 / 5}}\left|\nabla v_{n}\right|^{2} \leq C\left(k_{n}^{2}+1\right) \int_{B_{1} \backslash B_{1 / 2}}\left|v_{n}\right|^{2} .
$$

It follows from (2.24) and (2.25) that there exits $r \in(3 / 5,4 / 5)(r$ depends on $n)$ such that

$$
\int_{\partial B_{r}}\left|\nabla v_{n}\right|^{2}+\left(1+k_{n}^{2}\right) \int_{\partial B_{r}}\left|v_{n}\right|^{2} \rightarrow 0 \quad \text { as } n \rightarrow \infty .
$$

Since $\Delta v_{n}+k_{n}^{2}\left(1+i / k_{n}\right) v_{n}=0$ in $B_{1} \backslash B_{r}$, we have

$$
-\int_{B_{1} \backslash B_{r}}\left|\nabla v_{n}\right|^{2}+\left(k_{n}^{2}+i k_{n}\right) \int_{B_{1} \backslash B_{r}}\left|v_{n}\right|^{2}=\int_{\partial B_{r}} \partial_{r} v_{n} \bar{v}_{n}-\int_{\partial B_{1}} \partial_{r} v_{n} \bar{v}_{n} .
$$

A combination of (2.20), (2.22), (2.24), (2.26), and (2.27) yields

$$
\lim _{n \rightarrow \infty} \int_{B_{1} \backslash B_{4 / 5}}\left|\nabla v_{n}\right|^{2}=0
$$

From (2.24) and (2.28), we conclude that

$$
\lim _{n \rightarrow \infty}\left\|v_{n}\right\|_{H^{\frac{1}{2}\left(\partial B_{1}\right)}} \leq C \lim _{n \rightarrow \infty}\left\|v_{n}\right\|_{H^{1}\left(B_{1} \backslash B_{4 / 5}\right)}=0
$$


This is a contradiction to (2.20), and thus (2.19) holds under the additional assumption that $\varepsilon \leq \varepsilon_{0}$ for some fixed $0<\varepsilon_{0}$, sufficiently small.

It remains to prove (2.19) for $\varepsilon_{0}<\varepsilon<\tau$. In this case, we first prove that

$$
\left\|v_{\varepsilon}\right\|_{L^{2}\left(B_{5} \backslash B_{1}\right)} \leq C \max \{k, 1 / k\}\|g\|_{H^{-\frac{1}{2}}\left(\partial B_{1}\right)},
$$

by contradiction, and then we show that

$$
\left\|v_{\varepsilon}\right\|_{H^{1}\left(B_{5} \backslash B_{1}\right)} \leq C \max \{k, 1 / k\}\|g\|_{H^{-\frac{1}{2}}\left(\partial B_{1}\right)} .
$$

We note that since $k$ is bounded $\left(k<\tau / \varepsilon_{0}\right)$ and $\varepsilon$ is bounded away from zero (2.30) implies (2.19). In the argument by contradiction one may without loss of generality assume the $\varepsilon_{n}$ converge to $\varepsilon_{1}>0$. Thus the system (2.21) is asymptotically similar to the $2 \mathrm{~d}$ system (2.5), and so the argument of proof proceeds in the same fashion as in the two dimensional case presented above. The details are left to the reader.

We next provide some useful estimates for $\sigma_{1, \varepsilon}$ which is defined as follows:

$$
\sigma_{1, \varepsilon}:=\left(F_{\varepsilon}^{-1}\right)_{*} \sigma_{1, c}, \quad \text { in } B_{2} \backslash B_{\varepsilon} .
$$

Lemma 4. Assume $k_{\varepsilon} \geq c_{*} \varepsilon^{-1}$, for some fixed constant $c_{*}>0$. We have

$$
\left|\sigma_{1, \varepsilon}\right| \leq \frac{C_{1}}{\varepsilon^{d-1} k_{\varepsilon}^{2}} \quad \text { if } k<\frac{c_{*}}{2} \varepsilon^{-1}
$$

and,

$$
\left|\sigma_{1, \varepsilon}\right| \leq \frac{C_{2}}{\varepsilon^{d-1} k} \quad \text { and } \quad \Im\left(\sigma_{1, \varepsilon}\right) \geq \frac{c_{3} k}{\max \left\{k_{\varepsilon}^{4}, k^{4}\right\}} \quad \text { if } k \geq \frac{c_{*}}{2} \varepsilon^{-1}
$$

for some positive constants $C_{1}, C_{2}, c_{3}$ independent of $\varepsilon, k$ and $k_{\varepsilon}$ (but dependent on $c_{*}$ ).

Proof. We recall, by (1.3) and the fact that $\sigma_{N}=\sigma_{D}=1$,

$$
\sigma_{1, c}=\frac{1}{k_{\varepsilon}^{2}-k^{2}-i k}
$$

and therefore

$$
\Im\left(\sigma_{1, c}\right)=\frac{k}{\left(k_{\varepsilon}^{2}-k^{2}\right)^{2}+k^{2}}
$$

If $k<\frac{c_{*}}{2} \varepsilon^{-1}$ then it follows from (2.32) that

$$
\left|\sigma_{1, c}\right| \leq \frac{C}{k_{\varepsilon}^{2}}
$$

since $k_{\varepsilon}^{2}-k^{2}>3 k_{\varepsilon}^{2} / 4$. In this proof, $C$ denotes a positive constant independent of $\varepsilon, k$, and $k_{\varepsilon}$.

If $k \geq \frac{c_{*}}{2} \varepsilon^{-1}$, then it follows from (2.32) that

$$
\left|\sigma_{1, c}\right| \leq \frac{C}{k}
$$


and from (2.33) that

$$
\Im\left(\sigma_{1, c}\right) \geq \frac{C k}{\max \left\{k_{\varepsilon}^{4}, k^{4}\right\}}
$$

Since $\sigma_{1, \varepsilon}=\left(F_{\varepsilon}^{-1}\right)_{*} \sigma_{1, c}$, the estimates in this lemma are now a consequence of the fact that

$$
1 / C \leq \operatorname{det} D F_{\varepsilon}^{-1} \leq C \varepsilon^{-d+1}
$$

\section{Stability estimates in the time harmonic regime}

Let $\hat{u}_{c}(k, \cdot)$ be the Fourier transform of $u_{c}(\cdot, x)$ with respect to $t,{ }^{4}$ i.e.,

$$
\hat{u}_{c}(k, x):=\frac{1}{\sqrt{2 \pi}} \int_{-\infty}^{\infty} u_{c}(t, x) e^{i k t} d t .
$$

Then $\hat{u}_{c} \in H_{\text {loc }}^{1}\left(\mathbb{R}^{d}\right.$ ) (for a.e. $k>0$ ) is the unique outgoing solution to

$$
\operatorname{div}\left(A_{c} \nabla \hat{u}_{c}\right)+k^{2} \Sigma_{c} \hat{u}_{c}=-\hat{f}
$$

where $\left(A_{c}, \Sigma_{c}\right)$ is given in (1.4) (see Proposition 2 in Section 4).

Define $\tilde{u}_{\varepsilon}(k, x)=\hat{u}_{c}\left(k, F_{\varepsilon}(x)\right)$. Then $\tilde{u}_{\varepsilon} \in H_{l o c}^{1}\left(\mathbb{R}^{d}\right)$ is the unique outgoing solution to

$$
\operatorname{div}\left(A_{\varepsilon} \nabla \tilde{u}_{\varepsilon}\right)+k^{2} \Sigma_{\varepsilon} \tilde{u}_{\varepsilon}=-\hat{f}
$$

where

$$
A_{\varepsilon}, \Sigma_{\varepsilon}=\left\{\begin{array}{cl}
I, 1 & \text { in } \mathbb{R}^{d} \backslash B_{2}, \\
I, \sigma_{\varepsilon}(x):=1+\sigma_{1, \varepsilon}(x) & \text { in } B_{2} \backslash B_{\varepsilon}, \\
\frac{1}{\varepsilon^{d-2}} I, \frac{1}{\varepsilon^{d}}(1+i / k) & \text { in } B_{\varepsilon} \backslash B_{\varepsilon / 2}, \\
\frac{1}{\varepsilon^{d-2}} a(x / \varepsilon), \frac{1}{\varepsilon^{d}} \sigma(x / \varepsilon) & \text { in } B_{\varepsilon / 2},
\end{array}\right.
$$

and

$$
\sigma_{1, \varepsilon}=\left(F_{\varepsilon}^{-1}\right)_{*} \sigma_{1, c}
$$

In this section, we establish the stability for solutions to (3.1), (3.2) for quite general $\sigma_{1, \varepsilon}$; hence in the remainder of this section, we do not assume that $\sigma_{1, \varepsilon}$ is of the form (3.3), but only that it satisfies certain bounds. We recall that

$$
a \text { is bounded, uniformly elliptic, and } \sigma \in L^{\infty}\left(B_{1 / 2}\right) \text { with } \Im(\sigma) \geq 0 \text {. }
$$

The first result of this section concerns the small to moderate frequency regime.

\footnotetext{
${ }^{4}$ After extending $u_{c}$ by 0 for $t<0$.
} 
Lemma 5. Let $d=2$ or $3, \tau>0,0<\varepsilon, k<\tau$, and $g \in L^{2}\left(\mathbb{R}^{d}\right)$ with $\operatorname{supp} g \subset B_{4} \backslash B_{\varepsilon}$. Assume that

$$
\left\|\sigma_{1, \varepsilon}\right\|_{L^{\infty}\left(B_{2} \backslash B_{\varepsilon}\right)} \leq C_{0}, \quad \text { and } \quad \Im\left(\sigma_{1, \varepsilon}\right) \geq 0
$$

Let $v_{\varepsilon} \in H_{l o c}^{1}\left(\mathbb{R}^{d}\right)$ be the unique outgoing solution to

$$
\operatorname{div}\left(A_{\varepsilon} \nabla v_{\varepsilon}\right)+k^{2} \Sigma_{\varepsilon} v_{\varepsilon}=g \text { in } \mathbb{R}^{d} .
$$

There exists a positive constant $C$, depending only on $d, \tau$ and $C_{0}$, such that

$$
\left\|v_{\varepsilon}\right\|_{L^{2}\left(B_{5} \backslash B_{\varepsilon}\right)} \leq C \max \{1,1 / k\}\|g\|_{L^{2}} .
$$

Remark 1. In Lemma 5, the support of $g$ is assumed to be inside $B_{4} \backslash B_{\varepsilon}$ not $B_{4} \backslash B_{2}$, since $g$ will be of the form $-k^{2} \sigma_{1, \varepsilon} \hat{u}_{1, \varepsilon}$, when we apply this lemma in the proof of Theorem 2 . The blow up technique does not work for Lemma 5 due to the presence of $\sigma_{1, \varepsilon} \neq 0$ inside $B_{2} \backslash B_{\varepsilon}$. It is not essential that the support of $g$ be inside $B_{4} \backslash B_{\varepsilon}$, this could be replaced by $B_{M} \backslash B_{\varepsilon}$ for any $M>4$. The constant $C$ in the estimate would depend on $M$.

Proof. The proof is based on a contradiction argument, in which we use an argument of removable singularity. Suppose (3.7) does not hold. Then there exist $\left\{k_{n}\right\},\left\{\varepsilon_{n}\right\} \subset(0, \tau), \sigma_{1, n}, a_{n}, \sigma_{n}$, and $\left\{g_{n}\right\}$, $\operatorname{supp} g_{n} \subset B_{4} \backslash B_{\varepsilon_{n}}$, such that (3.5) holds for $\sigma_{1, n}, a_{n}$ and $\sigma_{n}$ satisfy (3.4), and

$$
\max \left\{1,1 / k_{n}\right\}\left\|g_{n}\right\|_{L^{2}} \rightarrow 0 \text { as } n \rightarrow \infty, \quad\left\|v_{n}\right\|_{L^{2}\left(B_{5} \backslash B_{\varepsilon_{n}}\right)}=1
$$

Here $v_{n} \in H_{l o c}^{1}\left(\mathbb{R}^{d}\right)$ is the unique outgoing solution to

$$
\operatorname{div}\left(A_{n} \nabla v_{n}\right)+k_{n}^{2} \Sigma_{n} v_{n}=g_{n} \text { in } \mathbb{R}^{d}
$$

where $A_{n}, \Sigma_{n}$ are defined in the same way as $A_{\varepsilon}, \Sigma_{\varepsilon}$ with $k, \varepsilon, \sigma_{1, \varepsilon}, a$, and $\sigma$ replaced by $k_{n}, \varepsilon_{n}, \sigma_{1, n}, a_{n}$, and $\sigma_{n}$. Using the outgoing radiation condition, as in (2.24), we obtain,

$$
\frac{k_{n}}{\varepsilon_{n}^{d}} \int_{B_{\varepsilon_{n}} \backslash B_{\varepsilon_{n} / 2}}\left|v_{n}\right|^{2} \leq \int_{\mathbb{R}^{d}}\left|g_{n}\right|\left|v_{n}\right| .
$$

Here we also used that $\Im\left(\sigma_{1, n}\right)$ and $\Im\left(\sigma_{n}\right)$ are non-negative. Since $\operatorname{supp} g_{n} \subset B_{4} \backslash B_{\varepsilon_{n}}$ and $\left\|v_{n}\right\|_{L^{2}\left(B_{5} \backslash B_{\varepsilon_{n}}\right)}=1$, the above inequality implies that

$$
\frac{k_{n}+1}{\varepsilon_{n}^{d-1}} \int_{B_{\varepsilon_{n}} \backslash B_{\varepsilon_{n} / 2}}\left|v_{n}\right|^{2} \leq 2 \varepsilon_{n} \max \left\{1,1 / k_{n}\right\}\left\|g_{n}\right\|_{L^{2}} .
$$

We have

$$
\Delta v_{n}+\frac{k_{n}^{2}}{\varepsilon_{n}^{2}}\left(1+i / k_{n}\right) v_{n}=0 \text { in } B_{\varepsilon_{n}} \backslash B_{\varepsilon_{n} / 2} .
$$

It follows from Caccioppoli's inequality that

$$
\int_{B_{4 \varepsilon_{n} / 5} \backslash B_{3 \varepsilon_{n} / 5}}\left|\nabla v_{n}\right|^{2} \leq \frac{C\left(k_{n}^{2}+1\right)}{\varepsilon_{n}^{2}} \int_{B_{\varepsilon_{n}} \backslash B_{\varepsilon_{n} / 2}}\left|v_{n}\right|^{2},
$$


and so

$$
\frac{\varepsilon_{n}}{\varepsilon_{n}^{d-2}\left(k_{n}+1\right)} \int_{B_{4 \varepsilon_{n} / 5} \backslash B_{3 \varepsilon_{n} / 5}}\left|\nabla v_{n}\right|^{2} \leq \frac{C\left(k_{n}+1\right)}{\varepsilon_{n}^{d-1}} \int_{B_{\varepsilon_{n}} \backslash B_{\varepsilon_{n} / 2}}\left|v_{n}\right|^{2} .
$$

In this proof, $C$ denotes a positive constant depending only on $d$ and $\tau$. From (3.9) and (3.10), we obtain

$$
\frac{\varepsilon_{n}}{\varepsilon_{n}^{d-2}\left(k_{n}+1\right)} \int_{B_{4 \varepsilon_{n} / 5} \backslash B_{3 \varepsilon_{n} / 5}}\left|\nabla v_{n}\right|^{2} \leq C \varepsilon_{n} \max \left\{1,1 / k_{n}\right\}\left\|g_{n}\right\|_{L^{2}} .
$$

A combination of (3.9) and (3.11) now yields

$$
\frac{1}{\varepsilon_{n}^{d-2}} \int_{B_{4 \varepsilon_{n} / 5} \backslash B_{3 \varepsilon_{n} / 5}}\left(\frac{k_{n}+1}{\varepsilon_{n}}\left|v_{n}\right|^{2}+\frac{\varepsilon_{n}}{k_{n}+1}\left|\nabla v_{n}\right|^{2}\right) \leq C \varepsilon_{n} \max \left\{1,1 / k_{n}\right\}\left\|g_{n}\right\|_{L^{2}} .
$$

It follows that for some $\alpha \in\left(3 \varepsilon_{n} / 5,4 \varepsilon_{n} / 5\right)$ ( $\alpha$ depends on $n$ ),

$$
\frac{1}{\varepsilon_{n}^{d-2}} \int_{\partial B_{\alpha}}\left(\frac{k_{n}+1}{\varepsilon_{n}}\left|v_{n}\right|^{2}+\frac{\varepsilon_{n}}{k_{n}+1}\left|\nabla v_{n}\right|^{2}\right) \leq C \max \left\{1,1 / k_{n}\right\}\left\|g_{n}\right\|_{L^{2}} \rightarrow 0 \quad \text { as } n \rightarrow \infty .
$$

Here we used (3.8) for the last convergence assertion. Multiplying the equation for $v_{n}$ by $\bar{v}_{n}$, and integrating on $B_{5} \backslash B_{\alpha}$, we have

$$
\begin{aligned}
& -\int_{B_{5} \backslash B_{\varepsilon_{n}}}\left|\nabla v_{n}\right|^{2}+k_{n}^{2} \int_{B_{5} \backslash B_{\varepsilon_{n}}}\left(1+\sigma_{1, n}\right)\left|v_{n}\right|^{2}-\frac{1}{\varepsilon_{n}^{d-2}} \int_{B_{\varepsilon_{n}} \backslash B_{\alpha}}\left|\nabla v_{n}\right|^{2} \\
& +\frac{k_{n}^{2}}{\varepsilon_{n}^{d}}\left(1+\frac{i}{k_{n}}\right) \int_{B_{\varepsilon_{n}} \backslash B_{\alpha}}\left|v_{n}\right|^{2}=\int_{B_{5}} g_{n} \bar{v}_{n}-\int_{\partial B_{5}} \partial_{r} v_{n} \bar{v}_{n}+\frac{1}{\varepsilon_{n}^{d-2}} \int_{\partial B_{\alpha}} \partial_{r} v_{n} \bar{v}_{n} .
\end{aligned}
$$

Here and in what follows, we extend $\sigma_{1, n}$ by 0 in $\mathbb{R}^{d} \backslash B_{2}$. Since $v_{n} \in H_{l o c}^{1}\left(\mathbb{R}^{d} \backslash B_{9 / 2}\right)$ is an outgoing solution to $\Delta v_{n}+k_{n}^{2} v_{n}=0$ in $\mathbb{R}^{d} \backslash B_{9 / 2}$, we have (see, e.g., Lemma 2)

$$
\left\|v_{n}\right\|_{H^{1}\left(B_{6} \backslash B_{9 / 2}\right)} \leq C\left\|v_{n}\right\|_{H^{1 / 2}\left(\partial B_{9 / 2}\right)},
$$

and so, by the standard theory of elliptic equations,

$$
\left\|v_{n}\right\|_{H^{1}\left(B_{6} \backslash B_{9 / 2}\right)} \leq C\left\|v_{n}\right\|_{L^{2}\left(B_{5} \backslash B_{\varepsilon_{n}}\right)} .
$$

Using (3.5), (3.8), (3.9), (3.12), and (3.14), in combination with (3.13), we now obtain

$$
\int_{B_{5} \backslash B_{\alpha}}\left|\nabla v_{n}\right|^{2} \leq C
$$

Define $u_{n} \in H^{1}\left(B_{\alpha}\right)$ as follows

$$
\Delta u_{n}=0 \text { in } B_{\alpha} \quad \text { and } \quad u_{n}=v_{n} \text { on } \partial B_{\alpha} .
$$

We derive from (3.8) and (3.12) that 


$$
\int_{B_{\alpha}}\left|\nabla u_{n}\right|^{2}+\left|u_{n}\right|^{2} \rightarrow 0 \text { as } n \rightarrow \infty
$$

Indeed, set $w_{n}(x)=u_{n}(\alpha x)$ for $x \in B_{1}$. Then

$$
\Delta w_{n}=0 \text { in } B_{1},
$$

and

$$
\left\|w_{n}\right\|_{H^{1}\left(\partial B_{1}\right)}^{2} \leq C\left(\alpha^{1-d} \int_{\partial B_{\alpha}}\left|u_{n}\right|^{2}+\alpha^{3-d} \int_{\partial B_{\alpha}}\left|\nabla u_{n}\right|^{2}\right) \leq C \max \left\{1,1 / k_{n}\right\}\left\|g_{n}\right\|_{L^{2}},
$$

where we used (3.12), and the fact that $3 \varepsilon_{n} / 5<\alpha<4 \varepsilon_{n} / 5$ for the last estimate. It follows that

$$
\int_{B_{1}}\left|\nabla w_{n}\right|^{2}+\left|w_{n}\right|^{2} \leq C \max \left\{1,1 / k_{n}\right\}\left\|g_{n}\right\|_{L^{2}}
$$

which in terms of $u_{n}$ yields

$$
\alpha^{-d} \int_{B_{\alpha}}\left|u_{n}\right|^{2}+\alpha^{2-d} \int_{B_{\alpha}}\left|\nabla u_{n}\right|^{2} \leq C \max \left\{1,1 / k_{n}\right\}\left\|g_{n}\right\|_{L^{2}} .
$$

The assertion (3.16) now follows from (3.8). Define

$$
V_{n}= \begin{cases}v_{n} & \text { in } \mathbb{R}^{d} \backslash B_{\alpha} \\ u_{n} & \text { in } B_{\alpha}\end{cases}
$$

We derive from (3.8), (3.9), (3.15), and (3.16) that

$$
\int_{B_{5}}\left|\nabla V_{n}\right|^{2}+\left|V_{n}\right|^{2} \leq C .
$$

It follows that (see, e.g., Lemma 2)

$$
\left\|V_{n}\right\|_{H^{1}\left(B_{R} \backslash B_{4}\right)} \leq C_{R}\left\|V_{n}\right\|_{H^{1 / 2}\left(\partial B_{4}\right)} \leq C_{R}\left\|V_{n}\right\|_{H^{1}\left(B_{5}\right)} \leq C_{R},
$$

for any $R \geq 5$, and as a consequence

$$
\left\|V_{n}\right\|_{H^{1}\left(B_{R}\right)} \leq C_{R}
$$

for all $R>0$. After extraction of a subsequence we may thus assume that $k_{n} \rightarrow k_{0} \geq 0, \varepsilon_{n} \rightarrow \varepsilon_{0} \geq 0$, $\alpha \rightarrow \alpha_{0}$ (recall that $\alpha$ depends on $\left.n\right), \sigma_{1, \varepsilon_{n}} \rightarrow \sigma_{1}$ weakly in $L^{2}\left(\sigma_{1}\right.$ satisfies (3.5)), and $V_{n} \rightarrow V$ weakly in $H_{l o c}^{1}\left(\mathbb{R}^{d}\right)$.

Suppose $k_{0}>0$. If $\varepsilon_{0}=0$ then $V$ is an outgoing solution to the equation

$$
\Delta V+k_{0}^{2}\left(1+\sigma_{1}\right) V=0 \text { in } \mathbb{R}^{d} \backslash\{0\} .
$$

Since $V \in H_{\text {loc }}^{1}\left(\mathbb{R}^{d}\right)$, it follows that

$$
\Delta V+k_{0}^{2}\left(1+\sigma_{1}\right) V=0 \text { in } \mathbb{R}^{d} .
$$


Therefore, $V=0$, and we have a contradiction to the fact that $\int_{B_{5}}|V|^{2}=\lim \int_{B_{5} \backslash B_{\varepsilon_{n}}}\left|V_{n}\right|^{2}=1$. Similarly, if $k_{0}>0$ and $\varepsilon_{0}>0$ (and thus $\alpha_{0}>0$ ), then $V$ is an outgoing solution to

$$
\Delta V+k_{0}^{2}\left(1+\sigma_{1}\right) V=0 \text { in } \mathbb{R}^{d} \backslash B_{\alpha_{0}} .
$$

It follows from (3.16) that $V=0$ in $B_{\alpha_{0}}$. Hence $\left.V\right|_{\mathbb{R}^{d} \backslash B_{\alpha_{0}}}$ is the unique outgoing solution to (3.17) with $V=0$ on $\partial B_{\alpha_{0}}$, and as a consequence $V=0$ in all of $\mathbb{R}^{d}$; we have also arrived at a contradiction.

This leaves the case $k_{0}=0$. We start by considering the case $\varepsilon_{0}>0$ (and thus $\alpha_{0}>0$ ). By Lemma 2, $V \in W^{1}\left(\mathbb{R}^{d} \backslash B_{\alpha_{0}}\right)$ is a solution to the equation

$$
\Delta V=0 \text { in } \mathbb{R}^{d} \backslash \bar{B}_{\alpha_{0}} .
$$

It follows from (3.16) that $V=0$ in $B_{\alpha_{0}}$, and thus $V$ is the unique solution to (3.18), with $V=0$ on $\partial B_{\alpha_{0}}$. Hence $V=0$ in $\mathbb{R}^{d} \backslash B_{\alpha_{0}}$, and as a consequence $V=0$ in $\mathbb{R}^{d}$, so we have arrived at a contradiction.

Finally we consider the case $k_{0}=\varepsilon_{0}=0$. By Lemma $2, V \in W^{1}\left(\mathbb{R}^{d}\right)$ is a solution to the equation

$$
\Delta V=0 \text { in } \mathbb{R}^{d} \backslash\{0\} .
$$

Since $V \in W^{1}\left(\mathbb{R}^{d}\right)$, it follows that

$$
\Delta V=0 \text { in } \mathbb{R}^{d}
$$

Thus $V=0$ in the case $d=3$, and we have arrived at a contradiction in three dimensions. In two dimensions, we can only at present conclude that $V$ is a constant, due to (3.20). We proceed to prove that $V=0$ in the case $d=2$ as well. Set

$$
\tilde{v}_{n}(x)=v_{n}\left(\varepsilon_{n} x\right) \text { for } x \in B_{1} \backslash B_{4 / 5} \text {. }
$$

From (3.8), (3.9), and (3.15), we have

$$
\left\|\tilde{v}_{n}\right\|_{H^{1 / 2}\left(\partial B_{1}\right)}^{2} \leq C\left(\int_{B_{1} \backslash B_{4 / 5}}\left|\nabla \tilde{v}_{n}\right|^{2}+\left|\tilde{v}_{n}\right|^{2}\right)=C\left(\int_{B_{\varepsilon} \backslash B_{4 \varepsilon / 5}}\left|\nabla v_{n}\right|^{2}+\varepsilon_{n}^{-2}\left|v_{n}\right|^{2}\right) \leq C .
$$

Since $\lim _{n \rightarrow \infty}\left\|\tilde{v}_{n}\right\|_{L^{2}\left(B_{1} \backslash B_{4 / 5}\right)}=0$ by (3.8) and (3.9), it follows from (3.21) that $\tilde{v}_{n} \rightarrow 0$ weakly in $H^{1}\left(B_{1} \backslash B_{4 / 5}\right)$, and thus

$$
\tilde{v}_{n} \rightarrow 0 \text { weakly in } H^{1 / 2}\left(\partial B_{1}\right) .
$$

Let $v_{1, n} \in H_{l o c}^{1}\left(\mathbb{R}^{2}\right)$ be the unique outgoing solution to

$$
\Delta v_{1, n}+k_{n}^{2} v_{1, n}=-k_{n}^{2} \sigma_{1, n} v_{n} \text { in } \mathbb{R}^{2} .
$$

Applying Lemma 1, the regularity theory of elliptic equations, and using (3.5) and (3.8), we have

$$
\frac{1}{k_{n}+1}\left\|\nabla^{2} v_{1, n}\right\|_{L^{2}\left(B_{5}\right)}+\left\|\nabla v_{1, n}\right\|_{L^{2}\left(B_{5}\right)}+\left(k_{n}+1\right)\left\|v_{1, n}\right\|_{L^{2}\left(B_{5}\right)} \leq C k_{n}^{2}\left(\left|\ln k_{n}\right|+1\right) .
$$

As a consequence of this and the fact that $k_{n} \rightarrow 0$,

$$
\left\|\nabla v_{1, n}\right\|_{L^{2}\left(B_{5}\right)}+\left\|v_{1, n}\right\|_{L^{\infty}\left(B_{5}\right)} \leq C k_{n}^{2}\left(\left|\ln k_{n}\right|+1\right) .
$$


By a rescaling (remember $d=2$ ) we get

$$
\left\|\nabla \tilde{v}_{1, n}\right\|_{L^{2}\left(B_{5}\right)}+\left\|\tilde{v}_{1, n}\right\|_{L^{\infty}\left(B_{5}\right)} \leq C k_{n}^{2}\left(\left|\ln k_{n}\right|+1\right)
$$

with $\tilde{v}_{1, n}(x)=v_{1, n}\left(\varepsilon_{n} x\right)$, and thus

$$
\left\|\tilde{v}_{1, n}\right\|_{H^{1 / 2}\left(\partial B_{1}\right)} \leq C k_{n}^{2}\left(\left|\ln k_{n}\right|+1\right) \rightarrow 0
$$

We define

$$
w_{n}=v_{n}-v_{1, n} \text { in } \mathbb{R}^{2} \backslash B_{\varepsilon_{n}},
$$

where $w_{n} \in H_{l o c}^{1}\left(\mathbb{R}^{2} \backslash B_{\varepsilon_{n}}\right)$ is the unique outgoing solution to

$$
\Delta w_{n}+k_{n}^{2} w_{n}=0 \text { in } \mathbb{R}^{2} \backslash B_{\varepsilon_{n}} \quad \text { and } \quad w_{n}=v_{n}-v_{1, n} \text { on } \partial B_{\varepsilon_{n}} .
$$

Set

$$
W_{n}(x)=w_{n}\left(\varepsilon_{n} x\right) \text { for } x \in \mathbb{R}^{2} \backslash B_{1} .
$$

Then $W_{n} \in H_{l o c}^{1}\left(\mathbb{R}^{2} \backslash B_{1}\right)$ is the unique outgoing solution to

$$
\Delta W_{n}+k_{n}^{2} \varepsilon_{n}^{2} W_{n}=0 \text { in } \mathbb{R}^{2} \backslash B_{1}, \quad \text { and } \quad W_{n}(x)=\tilde{v}_{n}(x)-\tilde{v}_{1, n}(x) \text { on } \partial B_{1} .
$$

Applying Lemma 2 for $W_{n}$ and using (3.22) and (3.24), we have $W_{n} \rightarrow 0$ weakly in $H_{l o c}^{1}\left(\mathbb{R}^{2} \backslash B_{1}\right)$, and by interior elliptic regularity estimates

$$
\left\|W_{n}\right\|_{H^{1 / 2}\left(\partial B_{2}\right)} \rightarrow 0 \text { as } n \rightarrow 0
$$

Applying Lemma 2 to $W_{n}$ again and rescaling, we obtain

$$
\left\|w_{n}\right\|_{L^{2}\left(B_{5} \backslash B_{4}\right)} \leq \frac{C\left|\ln k_{n}\right|}{\left|\ln \left(\varepsilon_{n} k_{n}\right)\right|}\left\|W_{n}\right\|_{H^{1 / 2}\left(\partial B_{2}\right)} \rightarrow 0 \text { as } n \rightarrow \infty .
$$

A combination of (3.23) and (3.25) yields that $v_{n} \rightarrow 0$ in $L^{2}\left(B_{5} \backslash B_{4}\right)$; it follows that $V=0$ in $B_{5} \backslash B_{4}$, and thus $V=0$ in all of $\mathbb{R}^{2}$ (since we already know it must be a constant). We have a contradiction, and the proof is complete.

The second result in this section deals with the moderate to high frequency regime.

Lemma 6. Let $d=2$ or $3,0<\varepsilon<1 / 2$, and $k>k_{0}>0$ for some constant $k_{0}$. Suppose $g \in L^{2}\left(\mathbb{R}^{d}\right)$ with $\operatorname{supp} g \subset B_{4} \backslash B_{\varepsilon}$ and let $v_{\varepsilon} \in H_{\text {loc }}^{1}\left(\mathbb{R}^{d}\right)$ be the unique outgoing solution to

$$
\operatorname{div}\left(A_{\varepsilon} \nabla v_{\varepsilon}\right)+k^{2} \Sigma_{\varepsilon} v_{\varepsilon}=g \text { in } \mathbb{R}^{d}
$$

Assume that

$$
\left\|\sigma_{1, \varepsilon}\right\|_{L^{\infty}}=\chi_{1} \quad \text { and } \quad \Im\left(\sigma_{1, \varepsilon}\right) \geq \chi_{2} \quad \text { a.e. in } \quad B_{2} \backslash B_{\varepsilon}
$$

for some $\chi_{1} \geq \chi_{2}>0$. There exist two positive constants $\lambda$ and $C$, independent of $k, \varepsilon, \chi_{1}, \chi_{2}$, and $g$ such that 
i) If $k \chi_{1} \leq \lambda$, then

$$
\int_{B_{5} \backslash B_{\varepsilon}}\left(\left|\nabla v_{\varepsilon}\right|^{2}+k^{2}\left|v_{\varepsilon}\right|^{2}\right) \leq C\left(k^{4}+1\right) \int_{\mathbb{R}^{d}}|g|^{2} .
$$

ii) If $k \chi_{1}>\lambda$, then

$$
\int_{B_{5} \backslash B_{\varepsilon}}\left(\left|\nabla v_{\varepsilon}\right|^{2}+k^{2}\left|v_{\varepsilon}\right|^{2}\right) \leq C\left(k^{4}+\frac{k^{2} \chi_{1}^{4}}{\chi_{2}^{2}}\right) \int_{\mathbb{R}^{d}}|g|^{2} .
$$

Remark 2. As in the previous lemma, it is not essential that the support of $g$ be inside $B_{4} \backslash B_{\varepsilon}$, this could be replaced by $B_{M} \backslash B_{\varepsilon}$ for any $M>4$. The constants in the estimates would depend on $M$. We also note that the estimate (3.28) is stronger than the estimate (3.29), since $k>k_{0}>0$. It thus follows immediately that

$$
\int_{B_{5} \backslash B_{\varepsilon}}\left(\left|\nabla v_{\varepsilon}\right|^{2}+k^{2}\left|v_{\varepsilon}\right|^{2}\right) \leq C\left(k^{4}+\frac{k^{2} \chi_{1}^{4}}{\chi_{2}^{2}}\right) \int_{\mathbb{R}^{d}}|g|^{2},
$$

for all $k>k_{0}>0$. These facts shall both be used in the proof of Theorem 2 .

Proof. The proof is inspired by [21]. To simplify notation we drop the subscript $\varepsilon$ from $v_{\varepsilon}$. Multiplying (3.26) by $\bar{v}$ and integrating on $B_{R}, R>1$, we obtain

$$
\int_{\partial B_{R}} \partial_{r} v \bar{v}-\int_{B_{R}}\left\langle A_{\varepsilon} \nabla v, \nabla \bar{v}\right\rangle+k^{2} \int_{B_{R}} \Sigma_{\varepsilon}|v|^{2}=\int_{B_{R}} g \bar{v} .
$$

Letting $R$ go to infinity, using the outgoing condition, and considering the imaginary part, we have

$$
k \limsup _{R \rightarrow \infty} \int_{\partial B_{R}}|v|^{2}+\frac{k}{\varepsilon^{d}} \int_{B_{\varepsilon} \backslash B_{\varepsilon / 2}}|v|^{2}+k^{2} \chi_{2} \int_{B_{2} \backslash B_{\varepsilon}}|v|^{2} \leq \int_{\mathbb{R}^{d}}|g||v| .
$$

Since $\Delta v+\frac{k^{2}}{\varepsilon^{2}} v+i \frac{k}{\varepsilon^{2}} v=0$ in $B_{\varepsilon} \backslash B_{\varepsilon / 2}$ and $k>k_{0}$, it follows from Caccioppoli's inequality that

$$
\int_{B_{4 \varepsilon / 5} \backslash B_{3 \varepsilon / 5}}|\nabla v|^{2} \leq \frac{C k^{2}}{\varepsilon^{2}} \int_{B_{\varepsilon} \backslash B_{\varepsilon / 2}}|v|^{2} .
$$

In this proof, $C$ denotes a positive constant independent of $\varepsilon, k, \chi_{1}, \chi_{2}$, and $g$. It follows from (3.30) and (3.31) that

$$
\int_{B_{4 \varepsilon / 5} \backslash B_{3 \varepsilon / 5}}|\nabla v|^{2}+\frac{k^{2}}{\varepsilon^{2}} \int_{B_{\varepsilon} \backslash B_{\varepsilon / 2}}|v|^{2} \leq C \varepsilon^{d-2} k \int_{\mathbb{R}^{d}}|g||v| .
$$

Thus there exists $t \in(3 \varepsilon / 5,4 \varepsilon / 5)$ such that

$$
\int_{\partial B_{t}}|\nabla v|^{2} \leq C \varepsilon^{d-3} k \int_{\mathbb{R}^{d}}|g||v| \quad \text { and } \quad \int_{\partial B_{t}}|v|^{2} \leq \frac{C \varepsilon^{d-1}}{k} \int_{\mathbb{R}^{d}}|g||v| \text {. }
$$


Applying [21, Lemma 2] with $\alpha=\varepsilon$ and $R>\beta \geq 5$ ( $\beta$ is a fixed constant which will be chosen later), we have $^{5}$

$$
\begin{aligned}
& \frac{1}{d-1} \int_{B_{\beta} \backslash B_{\varepsilon}}|\nabla v|^{2}+k^{2}|v|^{2} \leq F_{\beta}\left(\varepsilon, v_{+}\right)-F_{\beta}(R, v)+\frac{\beta(3-d)}{2} \int_{B_{R} \backslash B_{\beta}} \frac{|v|^{2}}{r^{3}} \\
& +C \int_{\mathbb{R}^{d}}|g|\left(\left|v^{\prime}\right|+|v|\right)+C \int_{B_{2} \backslash B_{\varepsilon}} k^{2} \chi_{1}\left(|v|\left|v^{\prime}\right|+|v|^{2}\right) .
\end{aligned}
$$

Here

$$
\begin{aligned}
F_{\beta}(r, v)= & -\frac{k^{2}}{2} \int_{\partial B_{r}} P_{*}(r)|v|^{2}-\frac{1}{2} \int_{\partial B_{r}} P_{*}(r)\left|v^{\prime}\right|^{2}+\frac{1}{2} \int_{\partial B_{r}} Q_{*}^{\prime}(r)|v|^{2} \\
& -\frac{1}{2} \int_{\partial B_{r}} Q_{*}(r)\left(|v|^{2}\right)^{\prime}+\frac{1}{2} \int_{\partial B_{r}} P_{*}(r)\left|\nabla_{\partial B_{r}} v\right|^{2},
\end{aligned}
$$

with

$$
P_{*}(r)=\left\{\begin{array}{l}
\frac{2 \beta}{d-1} \quad \text { if } r>\beta, \\
\frac{2 r}{d-1} \quad \text { if } 0<r<\beta,
\end{array} \quad \text { and } \quad Q_{*}(r)= \begin{cases}\frac{\beta}{r} & \text { if } r>\beta \\
1 & \text { if } 0<r<\beta\end{cases}\right.
$$

Note that

$$
\begin{gathered}
F_{\beta}(r, v)=F(r, v):=-\frac{k^{2} r}{d-1} \int_{\partial B_{r}}|v|^{2}-\frac{r}{d-1} \int_{\partial B_{r}}\left|v^{\prime}\right|^{2} \\
-\frac{1}{2} \int_{\partial B_{r}}\left(|v|^{2}\right)^{\prime}+\frac{r}{d-1} \int_{\partial B_{r}}\left|\nabla_{\partial B_{r}} v\right|^{2},
\end{gathered}
$$

for $0<r<\beta$ (where $F$ is independent of $\beta$ ). Since $P_{*}(r)=\frac{2 r}{d-1}$ and $Q_{*}(r)=1$ for $0<r<\beta$,

$$
\Re \int_{B_{\varepsilon} \backslash B_{t}}\left(\Delta v+k^{2} v\right)\left[\frac{2 r}{d-1} \bar{v}_{r}+\bar{v}\right]=\int_{B_{\varepsilon} \backslash B_{t}} \Re\left[\left(\Delta v+k^{2} v\right)\left(\frac{2}{d-1} x \cdot \nabla \bar{v}+\bar{v}\right)\right] .
$$

We have ${ }^{6}$

$$
\begin{aligned}
& \Re\left[\left(\Delta v+k^{2} v\right)\left(\frac{2}{d-1} x \cdot \nabla \bar{v}+\bar{v}\right)\right]=-\frac{1}{d-1}\left(|\nabla v|^{2}+k^{2}|v|^{2}\right) \\
& \quad+\Re \nabla \cdot\left[\frac{2}{d-1} \nabla v(x \cdot \nabla \bar{v})-\frac{1}{d-1} x|\nabla v|^{2}+\nabla v \bar{v}+\frac{k^{2}}{d-1} x|v|^{2}\right] .
\end{aligned}
$$

Integrating over the domain $B_{\varepsilon} \backslash B_{t}$, we obtain:

\footnotetext{
5 This inequality is a variant of an inequality due to Morawetz and Ludwig [15] (see also [24]).

6 This is the "Rellich" identity which originates from $[15,22,25]$.
} 


$$
\begin{aligned}
& \Re \int_{B_{\varepsilon} \backslash B_{t}}\left(\Delta v+k^{2} v\right)\left[\frac{2 r}{d-1} \bar{v}_{r}+\bar{v}\right]+\frac{1}{d-1} \int_{B_{\varepsilon} \backslash B_{t}}\left(|\nabla v|^{2}+k^{2}|v|^{2}\right) \\
& =\Re \int_{\partial B_{\varepsilon}}\left(\frac{2 \varepsilon}{d-1}\left|v_{r}\right|^{2}-\frac{\varepsilon}{d-1}|\nabla v|^{2}+v_{r} \bar{v}+\frac{k^{2} \varepsilon}{d-1}|v|^{2}\right) \\
& \quad-\Re \int_{\partial B_{t}}\left(\frac{2 t}{d-1}\left|v_{r}\right|^{2}-\frac{t}{d-1}|\nabla v|^{2}+v_{r} \bar{v}+\frac{k^{2} t}{d-1}|v|^{2}\right) .
\end{aligned}
$$

It follows that

$$
\begin{aligned}
& \frac{1}{\varepsilon^{d-2}(d-1)} \int_{B_{\varepsilon} \backslash B_{t}}|\nabla v|^{2}+k^{2}|v|^{2} \leq-\frac{1}{\varepsilon^{d-2}} F\left(\varepsilon, v_{-}\right)+\frac{1}{\varepsilon^{d-2}} F(t, v) \\
& +\frac{C k^{2}}{\varepsilon^{d}} \int_{B_{\varepsilon} \backslash B_{t}}\left(\varepsilon|v|\left|v^{\prime}\right|+|v|^{2}\right) .
\end{aligned}
$$

Adding (3.33) and (3.36), we obtain

$$
\begin{aligned}
& \int_{B_{\beta} \backslash B_{\varepsilon}}\left(|\nabla v|^{2}+k^{2}|v|^{2}\right)+\frac{1}{\varepsilon^{d-2}} \int_{B_{\varepsilon} \backslash B_{t}}\left(|\nabla v|^{2}+k^{2}|v|^{2}\right) \\
& \leq(d-1)\left(F\left(\varepsilon, v_{+}\right)-F_{\beta}(R, v)-\frac{1}{\varepsilon^{d-2}} F\left(\varepsilon, v_{-}\right)+\frac{1}{\varepsilon^{d-2}} F(t, v)\right) \\
& \quad+\frac{C k^{2}}{\varepsilon^{d}} \int_{B_{\varepsilon} \backslash B_{t}}\left(\varepsilon|v|\left|v^{\prime}\right|+|v|^{2}\right)+\frac{\beta(3-d)(d-1)}{2} \int_{B_{R} \backslash B_{\beta}} \frac{|v|^{2}}{r^{3}} \\
&+C \int_{\mathbb{R}^{d}}|g|\left(\left|v^{\prime}\right|+|v|\right)+C \int_{B_{2} \backslash B_{\varepsilon}} k^{2} \chi_{1}\left(|v|\left|v^{\prime}\right|+|v|^{2}\right) .
\end{aligned}
$$

We next estimate the first and second lines of the RHS of (3.37). We start with the first line. Using the outgoing condition, we have

$$
\limsup _{R \rightarrow \infty}-F_{\beta}(R, v) \leq C \beta k^{2} \limsup _{R \rightarrow \infty} \int_{\partial B_{R}}|v|^{2},
$$

which implies, by (3.30),

$$
\limsup _{R \rightarrow \infty}-F_{\beta}(R, v) \leq C \beta k \int_{\mathbb{R}^{d}}|g||v| .
$$

We claim that

$$
F\left(\varepsilon, v_{+}\right)-\frac{1}{\varepsilon^{d-2}} F\left(\varepsilon, v_{-}\right) \leq C k^{2} \int_{\partial B_{\varepsilon}}|v|^{2} .
$$

In fact, if $d=2$ then there is nothing to prove since $v_{+}=v_{-}$and $\partial_{r} v_{+}=\partial_{r} v_{-}$on $\partial B_{\varepsilon}$. Assume $d=3$. Since $v_{+}=v_{-}$on $\partial B_{\varepsilon}$, and $\varepsilon<1$ we get, by (3.34), 


$$
\begin{aligned}
F\left(\varepsilon, v_{+}\right)-\frac{1}{\varepsilon} F\left(\varepsilon, v_{-}\right) \leq & \frac{k^{2}(1-\varepsilon)}{2} \int_{\partial B_{\varepsilon}}|v|^{2}-\frac{\varepsilon}{2} \int_{\partial B_{\varepsilon}}\left|v_{+}^{\prime}\right|^{2}+\frac{1}{2} \int_{\partial B_{\varepsilon}}\left|v_{-}^{\prime}\right|^{2} \\
& -\frac{1}{2} \int_{\partial B_{\varepsilon}}\left(\left|v_{+}\right|^{2}\right)^{\prime}+\frac{1}{2 \varepsilon} \int_{\partial B_{\varepsilon}}\left(\left|v_{-}\right|^{2}\right)^{\prime}
\end{aligned}
$$

Using the fact that $v_{+}^{\prime}=(1 / \varepsilon) v_{-}^{\prime}$ (and $\varepsilon<1$ ), claim (3.39) follows from (3.40). We next estimate the RHS of (3.39). We have

$$
\int_{\partial B_{\varepsilon}}|v|^{2}-(\varepsilon / t)^{d-1} \int_{\partial B_{t}}|v|^{2} \leq C\left(\int_{B_{\varepsilon} \backslash B_{t}}|\nabla v|^{2}\right)^{1 / 2}\left(\int_{B_{\varepsilon} \backslash B_{t}}|v|^{2}\right)^{1 / 2} .
$$

This implies

$$
\int_{\partial B_{\varepsilon}}|v|^{2}-(\varepsilon / t)^{d-1} \int_{\partial B_{t}}|v|^{2} \leq \frac{C c}{k^{2} \varepsilon^{d-2}} \int_{B_{\varepsilon} \backslash B_{t}}|\nabla v|^{2}+\frac{C k^{2} \varepsilon^{d-2}}{c} \int_{B_{\varepsilon} \backslash B_{t}}|v|^{2},
$$

for some small positive constant $c$, which will be chosen later. A combination of (3.39) and (3.41) yields

$$
\begin{aligned}
& F\left(\varepsilon, v_{+}\right)-\frac{1}{\varepsilon^{d-2}} F\left(\varepsilon, v_{-}\right) \\
& \quad \leq C k^{2}\left[\frac{c}{k^{2} \varepsilon^{d-2}} \int_{B_{\varepsilon} \backslash B_{t}}|\nabla v|^{2}+\frac{k^{2} \varepsilon^{d-2}}{c} \int_{B_{\varepsilon} \backslash B_{t}}|v|^{2}+\int_{\partial B_{t}}|v|^{2}\right] .
\end{aligned}
$$

From (3.30) and (3.32), we have

$$
\frac{k^{4} \varepsilon^{d-2}}{c} \int_{B_{\varepsilon} \backslash B_{t}}|v|^{2}+k^{2} \int_{\partial B_{t}}|v|^{2} \leq C\left(k^{3} \varepsilon^{2 d-2}+k \varepsilon^{d-1}\right) \int_{\mathbb{R}^{d}}|g| v \mid .
$$

It follows from (3.42) and (3.43), by choosing $c$ sufficiently small, that

$$
F\left(\varepsilon, v_{+}\right)-\frac{1}{\varepsilon^{d-2}} F\left(\varepsilon, v_{-}\right) \leq C\left(k^{3} \varepsilon^{2 d-2}+k \varepsilon^{d-1}\right) \int_{\mathbb{R}^{d}}|g||v|+\frac{1}{3 \varepsilon^{d-2}} \int_{B_{\varepsilon} \backslash B_{t}}|\nabla v|^{2} .
$$

We also have, by (3.34),

$$
F(t, v) \leq C \int_{\partial B_{t}}|v||\nabla v|+\varepsilon|\nabla v|^{2} \leq C\left(\varepsilon \int_{\partial B_{t}}|\nabla v|^{2}+\frac{1}{\varepsilon} \int_{\partial B_{t}}|v|^{2}\right)
$$

which, by (3.32), implies

$$
\frac{1}{\varepsilon^{d-2}} F(t, v) \leq C k \int_{\mathbb{R}^{d}}|g||v|
$$

Here we used that $k \geq k_{0}>0$. Combining (3.38) with (3.44) and (3.45), we reach the following estimate for the first line of the RHS of (3.37) 


$$
\begin{gathered}
F\left(\varepsilon, v_{+}\right)+\limsup _{R \rightarrow \infty}-F_{\beta}(R, v)-\frac{1}{\varepsilon^{d-2}} F\left(\varepsilon, v_{-}\right)+\frac{1}{\varepsilon^{d-2}} F(t, v) \\
\quad \leq C\left(k^{3} \varepsilon^{2 d-2}+k \varepsilon^{d-1}+\beta k\right) \int_{\mathbb{R}^{d}}|g||v|+\frac{1}{3 \varepsilon^{d-2}} \int_{B_{\varepsilon} \backslash B_{t}}|\nabla v|^{2} .
\end{gathered}
$$

Here we also used that $\beta \geq 5$. We next estimate the second line of the RHS of (3.37). For that purpose

$$
\frac{k^{2}}{\varepsilon^{d}} \int_{B_{\varepsilon} \backslash B_{t}}\left(\varepsilon|v|\left|v^{\prime}\right|+|v|^{2}\right) \leq \int_{B_{\varepsilon} \backslash B_{t}}\left(\left[\frac{k^{4}}{c \varepsilon^{d}}+\frac{k^{2}}{\varepsilon^{d}}\right]|v|^{2}+\frac{c}{\varepsilon^{d-2}}\left|v^{\prime}\right|^{2}\right)
$$

for $c>0$. Using (3.30) and choosing $c$ sufficiently small, we have

$$
C \frac{k^{2}}{\varepsilon^{d}} \int_{B_{\varepsilon} \backslash B_{t}}\left(\varepsilon|v|\left|v^{\prime}\right|+|v|^{2}\right) \leq C\left(k^{3}+k\right) \int_{B_{\varepsilon} \backslash B_{t}}|g||v|+\frac{1}{3 \varepsilon^{d-2}} \int_{B_{\varepsilon} \backslash B_{t}}\left|v^{\prime}\right|^{2} .
$$

On the other hand, using $[21,(2.25)-(2.26)]$, we have for $d=2$,

$$
\int_{B_{R} \backslash B_{\beta}} \frac{|v|^{2}}{r^{3}} \leq C \int_{\beta}^{\infty} \frac{1}{r^{3}} d r \int_{\partial B_{\beta}}|v|^{2} \leq \frac{C}{\beta^{2}} \int_{\partial B_{\beta}}|v|^{2} \leq \frac{C}{\beta^{2}} \int_{B_{5} \backslash B_{4}}|v|^{2} .
$$

From (3.47) and (3.48), we reach the following estimate for the second line of the RHS of (3.37)

$$
\begin{aligned}
& \frac{C k^{2}}{\varepsilon^{d}} \int_{B_{\varepsilon} \backslash B_{t}}\left(\varepsilon|v|\left|v^{\prime}\right|+|v|^{2}\right)+\frac{\beta(3-d)(d-1)}{2} \int_{B_{R} \backslash B_{5}} \frac{|v|^{2}}{r^{3}} \\
& \leq C\left(k^{3}+k\right) \int_{B_{\varepsilon} \backslash B_{t}}|g||v|+\frac{1}{3 \varepsilon^{d-2}} \int_{B_{\varepsilon} \backslash B_{t}}\left|v^{\prime}\right|^{2}+\frac{C}{\beta} \int_{B_{5} \backslash B_{4}}|v|^{2} .
\end{aligned}
$$

A combination of (3.37), (3.46) and (3.49) yields (by taking a "limit" of large $R$ )

$$
\begin{aligned}
& \int_{B_{\beta} \backslash B_{\varepsilon}}\left(|\nabla v|^{2}+k^{2}|v|^{2}\right)+\frac{1}{\varepsilon^{d-2}} \int_{B_{\varepsilon} \backslash B_{t}}\left(|\nabla v|^{2}+k^{2}|v|^{2}\right) \\
& \leq C\left(k^{3}+\beta k\right) \int_{\mathbb{R}^{d}}|g||v|+C \int_{\mathbb{R}^{d}}|g|\left|v^{\prime}\right|+C \int_{B_{2} \backslash B_{\varepsilon}} k^{2} \chi_{1}\left(\left|v \| v^{\prime}\right|+|v|^{2}\right),
\end{aligned}
$$

for $\beta$ sufficiently large. Here we used the fact that $0<\varepsilon<1 / 2$ and $k \geq k_{0}>0$.

Case 1: $k \chi_{1} \leq \lambda$. It follows from (3.50) that

$$
\int_{B_{5} \backslash B_{\varepsilon}}\left(|\nabla v|^{2}+k^{2}|v|^{2}\right)+\frac{1}{\varepsilon^{d-2}} \int_{B_{\varepsilon} \backslash B_{t}}\left(|\nabla v|^{2}+k^{2}|v|^{2}\right) \leq C\left(k^{4}+1\right) \int_{\mathbb{R}^{d}}|g|^{2},
$$

since

$$
\left(k^{3}+k\right)|g||v| \leq\left(k^{2}+1\right)(k+1)|g||v| \leq \frac{1}{c}\left(k^{2}+1\right)^{2}|g|^{2}+c(k+1)^{2}|v|^{2},
$$

and 


$$
|g|\left|v^{\prime}\right| \leq \frac{1}{c}|g|^{2}+c\left|v^{\prime}\right|^{2}
$$

In (3.51) we have absorbed the remaining terms of the RHS of (3.50) by the LHS (by taking $c$ sufficiently small) since $\lambda$ can also be chosen sufficiently small.

Case $2: k \chi_{1} \geq \lambda$. It follows from (3.50) that

$$
\int_{B_{5} \backslash B_{\varepsilon}}|\nabla v|^{2}+k^{2}|v|^{2}+\frac{1}{\varepsilon^{d-2}} \int_{B_{\varepsilon} \backslash B_{t}}|\nabla v|^{2}+k^{2}|v|^{2} \leq C\left(k^{4}+\frac{k^{2} \chi_{1}^{4}}{\chi_{2}^{2}}\right) \int_{\mathbb{R}^{d}}|g|^{2},
$$

since

$$
k^{2} \chi_{1}\left(|v|\left|v^{\prime}\right|+|v|^{2}\right) \leq c\left|v^{\prime}\right|^{2}+C k^{4} \chi_{1}^{2}|v|^{2}
$$

and, by (3.30),

$$
k^{4} \chi_{1}^{2} \int_{B_{2} \backslash B_{\varepsilon}}|v|^{2} \leq \frac{k^{2} \chi_{1}^{2}}{\chi_{2}} \int_{\mathbb{R}^{d}}|g||v| \leq \frac{C k^{2} \chi_{1}^{4}}{\chi_{2}^{2}} \int_{\mathbb{R}^{d}}|g|^{2}+c \int_{B_{4} \backslash B_{\varepsilon}} k^{2}|v|^{2} .
$$

Here we also used (3.52) and (3.53) to treat the remaining terms of the RHS of (3.50) in the same fashion as before. The proof is complete.

\section{Weak solutions and the well-posedness of the non-local wave equations}

In this section, we first introduce the notion of weak solutions for the system (1.6) and establish the well-posedness of these. We then outline a proof of the fact that the Fourier transform in time of these solutions solve a corresponding "outgoing" Helmholtz problem for almost every frequency. We start with:

Definition 1. Let $d=2$ or $d=3$. We say a function

$$
u \in L^{\infty}\left([0, \infty) ; H^{1}\left(\mathbb{R}^{d}\right)\right) \text { with } \partial_{t} u \in L^{\infty}\left([0, \infty) ; L^{2}\left(\mathbb{R}^{d}\right)\right)
$$

is a weak solution to $(1.6)$ provided $u(0, x)=0$ in $\mathbb{R}^{d}$ and for all $t>0$

$$
\begin{aligned}
& -\int_{0}^{t} \int_{\mathbb{R}^{d}} \Sigma_{1, c}(x) \frac{\partial}{\partial s} u(s, x) \frac{\partial}{\partial s} v(s, x) d x d s+\int_{0}^{t} \int_{\mathbb{R}^{d}}\left\langle A_{c}(x) \nabla u(s, x), \nabla v(s, x)\right\rangle d x d s \\
& \quad+\int_{0}^{t} \int_{B_{1} \backslash B_{1 / 2}} \Sigma_{2, c}(x) \frac{\partial}{\partial s} u(s, x) v(s, x) d x d s+\int_{0}^{t} \int_{B_{2} \backslash B_{1}} G * \partial_{s} u(s, x) v(s, x) d x d s \\
& =\int_{0}^{t} \int_{\mathbb{R}^{d}} f(s, x) v(s, x) d x d s,
\end{aligned}
$$

for any $v \in L^{\infty}\left([0, \infty) ; H^{1}\left(\mathbb{R}^{d}\right)\right)$ with $\partial_{t} v \in L^{\infty}\left([0, \infty) ; L^{2}\left(\mathbb{R}^{d}\right)\right)$ and $v(t, x)=0$.

Note that $u \in C^{0}\left([0, \infty) ; L^{2}\left(\mathbb{R}^{d}\right)\right)$ and so the initial condition $u(0, x)=0$ makes sense, also note that the initial condition $u_{t}(0, x)=0$ is well-defined in a weak sense. It is clear that if $u \in C^{2}\left([0,+\infty) \times \mathbb{R}^{d}\right)$ is a 
weak solution in the sense defined above, then it is a classical solution to (1.6). Our definition is motivated by the standard definition of weak solutions to the wave equation.

The well-posedness of weak solutions to (1.6) is given by the following:

Proposition 1. Let $d=2$ or $d=3$, and let $f \in L^{\infty}\left([0, \infty) \times L^{2}\left(\mathbb{R}^{d}\right)\right)$ with compact support in $[0, \infty) \times \mathbb{R}^{d}$. Then there exists a unique $u \in L^{\infty}\left([0, \infty) ; H^{1}\left(\mathbb{R}^{d}\right)\right)$ with $\partial_{t} u \in L^{\infty}\left([0, \infty) ; L^{2}\left(\mathbb{R}^{d}\right)\right)$ which is a weak solution to (1.6). Moreover,

$$
E(t, u) \leq C t\|f\|_{L^{2}\left([0, t] \times \mathbb{R}^{d}\right)}^{2}, \text { for a.e. } t>0 .
$$

Here $C$ is a positive constant depending on $\Lambda$ and $\varepsilon$, but independent of $f$ and $t$, and

$$
E(t, u):=\frac{1}{2} \int_{\mathbb{R}^{d}}\left(\Sigma_{1, c}\left|\partial_{t} u(t, x)\right|^{2}+\left\langle A_{c} \nabla u(t, x), \nabla u(t, x)\right\rangle\right) d x .
$$

The proof is based on a standard Galerkin approach, as part of which we derive a non-trivial energy estimate. Similar ideas were used in [19].

Proof. We first establish the existence of a weak solution by an approximate (Galerkin) approach. Let $\left(\varphi_{j}\right)_{j=1}^{\infty} \subset C_{c}^{\infty}\left(\mathbb{R}^{d}\right)$ be an orthonormal basis in $H^{1}\left(\mathbb{R}^{d}\right)$. For $m \in \mathbb{N}$, consider $u_{m}$ of the form

$$
u_{m}=\sum_{j=1}^{m} d_{m, j}(t) \varphi_{j}(x), d_{m, j} \in C^{2}([0, \infty))
$$

satisfying

$$
\begin{aligned}
& \frac{d^{2}}{d s^{2}} \int_{\mathbb{R}^{d}} \Sigma_{1, c}(x) u_{m}(s, x) \varphi_{j}(x) d x+\int_{\mathbb{R}^{d}}\left\langle A_{c} \nabla u_{m}(s, x), \nabla \varphi_{j}(x)\right\rangle d x \\
& \quad+\frac{d}{d s} \int_{B_{1} \backslash B_{1 / 2}} \Sigma_{2, c}(x) u_{m}(s, x) \varphi_{j}(x) d x+\int_{B_{2} \backslash B_{1}} G * \partial_{s} u_{m}(s, x) \varphi_{j}(x) d x \\
& \quad=\int_{\mathbb{R}^{d}} f(s, x) \varphi_{j}(x) d x,
\end{aligned}
$$

for $j=1, \ldots, m$, and

$$
d_{m, j}(0)=d_{m, j}^{\prime}(0)=0 \quad \text { for } j=1, \ldots, m .
$$

Since $\left(\varphi_{j}\right)_{j}$ are linearly independent, the $(n \times n)$ matrix $M$ given by $M_{i, j}=\left\langle\varphi_{i}, \varphi_{j}\right\rangle_{L^{2}\left(\mathbb{R}^{d}\right)}$ is invertible. Therefore, the existence and uniqueness of $u_{m}$ follow by a standard argument, for example, one can use the theory of Volterra equations (see, e.g., [2, Theorem 2.1.1]).

We now derive an estimate for $u_{m}$. Multiplying (4.5) by $d_{m, j}^{\prime}(s)$, summing up with respect to $j$, integrating on $[0, t]$ with respect to $s$, and using (4.6) we obtain

$$
\begin{aligned}
& E\left(t, u_{m}\right)+\int_{0}^{t} \int_{B_{2} \backslash B_{1}} G * \partial_{s} u_{m}(s, x) \partial_{s} u_{m}(s, x) d x d s+\int_{0}^{t} \int_{B_{1} \backslash B_{1 / 2}} \Sigma_{2, c}\left|\partial_{s} u_{m}\right|^{2} d s d x \\
& =\int_{0}^{t} \int_{\mathbb{R}^{d}} f(s, x) \partial_{s} u_{m}(s, x) d x d s .
\end{aligned}
$$


We claim that

$$
\int_{0}^{t} \int_{B_{2} \backslash B_{1}} G * \partial_{s} u_{m}(s, x) \partial_{s} u_{m}(s, x) d x d s \geq 0 \quad \text { for a.e. } t>0 .
$$

Indeed, define

$$
U(s, x)=\left\{\begin{array}{cl}
\partial_{s} u_{m}(s, x) & \text { if } 0<s<t \\
0 & \text { if } s \geq t
\end{array}\right.
$$

and extend $U$ by zero for $s \leq 0$. Then

$$
\begin{aligned}
\int_{0}^{t} \int_{B_{2} \backslash B_{1}} G * \partial_{s} u_{m}(s, x) \partial_{s} u_{m}(s, x) d x d s & =\int_{-\infty}^{\infty} \int_{B_{2} \backslash B_{1}} G * U U d x d s \\
& =\int_{-\infty}^{\infty} \int_{B_{2} \backslash B_{1}} \widehat{G * U} \overline{\widehat{U}} d x d k=2 \Re \int_{0}^{\infty} \int_{B_{2} \backslash B_{1}} \widehat{G * U} \overline{\widehat{U}} d x d k \\
= & 2 \int_{0}^{\infty} \int_{B_{2} \backslash B_{1}} \Re(\widehat{G})|\widehat{U}|^{2} d x d k=2 \int_{0}^{\infty} \int_{B_{2} \backslash B_{1}} \frac{k^{2}}{\left(k_{\varepsilon}^{2}-k^{2}\right)^{2}+k^{2}}|\widehat{U}|^{2} d x d k \geq 0
\end{aligned}
$$

by the definition of $G$. This establishes (4.8). From (4.7) and (4.8), we arrive at

$$
E\left(t, u_{m}\right) \leq \int_{0}^{t} \int_{\mathbb{R}^{d}} f(s, x) \partial_{s} u_{m}(s, x) d x d s
$$

It follows from (4.9) that

$$
E\left(t, u_{m}\right) \leq\left(\int_{0}^{t} \int_{\mathbb{R}^{d}}\left|\partial_{s} u_{m}(s, x)\right|^{2} d x d s\right)^{1 / 2}\left(\int_{0}^{t} \int_{\mathbb{R}^{d}}|f(s, x)|^{2} d x d s\right)^{1 / 2}
$$

which implies

$$
E\left(t, u_{m}\right) \leq C\left(\int_{0}^{t} E\left(s, u_{m}\right) d s\right)^{1 / 2}\left(\int_{0}^{t} \int_{\mathbb{R}^{d}}|f(s, x)|^{2} d x d s\right)^{1 / 2} .
$$

Here and in the remainder of this proof, $C$ denotes a positive constant which depends on $\varepsilon$ and $\Lambda$, but is independent of $f, t$, and $m$. We derive from (4.10) that

$$
E\left(t, u_{m}\right) \leq C t \int_{0}^{t} \int_{\mathbb{R}^{d}}|f(s, x)|^{2} d x d s
$$

Hence, for any fixed $T>0$, there exists a subsequence of $\left(u_{m}\right)$ (which is also denoted by $u_{m}$ for notational ease) such that $u_{m} \rightarrow u$ weakly star in $L^{\infty}\left([0, T], H^{1}\left(\mathbb{R}^{d}\right)\right)$ and $\partial_{t} u_{m} \rightarrow \partial_{t} u$ weakly star in $L^{\infty}\left([0, T], L^{2}\left(\mathbb{R}^{d}\right)\right)$. It is clear that $u(0, x)=\partial_{t} u(0, x)=0$, and that $u$ satisfies (4.1) for any $v$ of the 
form $v(s, x)=\varphi_{j}(x) \psi(s), \psi \in C^{1}([0, \infty), \psi(t)=0$. By a standard linearity and approximation argument it follows that $u$ satisfies $(4.1)$ for any $v \in L^{\infty}\left([0, \infty), H^{1}\left(\mathbb{R}^{d}\right)\right)$ with $\partial_{t} v \in L^{\infty}\left([0, \infty), L^{2}\left(\mathbb{R}^{d}\right)\right)$ and $v(t, x)=0$. In other words, $u$ is a weak solution to (1.6). To see that $u$ is unique, it suffices to prove that if $w \in L^{\infty}\left([0, T], H^{1}\left(\mathbb{R}^{d}\right)\right)$, with $\partial_{t} w \in L^{\infty}\left([0, T], L^{2}\left(\mathbb{R}^{d}\right)\right), w(0, x)=\partial_{t} w(0, x)=0$, and $w$ satisfies $(4.1)$ with $f=0$ then $w$ is identically zero. We have

$$
\begin{aligned}
& -\int_{0}^{t} \int_{\mathbb{R}^{d}} \Sigma_{1, c}(x) \partial_{s} w(s, x) \partial_{s} v(s, x) d x d s+\int_{0}^{t} \int_{\mathbb{R}^{d}} A_{c}(x) \nabla w(s, x) \nabla v(s, x) d x d s \\
& \quad+\int_{0}^{t} \int_{B_{1} \backslash B_{1 / 2}} \Sigma_{2, c}(x) \partial_{s} w(s, x) v(s, x) d x d s+\int_{0}^{t} \int_{B_{2} \backslash B_{1}} G * \partial_{s} w(s, x) v(s, x) d x d s=0,
\end{aligned}
$$

for all $v \in L^{\infty}\left([0, \infty), H^{1}\left(\mathbb{R}^{d}\right)\right)$ with $\partial_{t} v \in L^{\infty}\left([0, \infty), L^{2}\left(\mathbb{R}^{d}\right)\right)$ and $v(t, x)=0$. After integration by parts, this implies

$$
\begin{aligned}
& -\int_{0}^{t} \int_{\mathbb{R}^{d}} \Sigma_{1, c}(x) \partial_{s} w(s, x) \partial_{s} v(s, x) d x d s+\int_{0}^{t} \int_{\mathbb{R}^{d}} A_{c}(x) \nabla w(s, x) \nabla v(s, x) d x d s \\
& \quad-\int_{0}^{t} \int_{B_{1} \backslash B_{1 / 2}} \Sigma_{2, c}(x) w(s, x) \partial_{s} v(s, x) d x d s-\int_{0}^{t} \int_{B_{2} \backslash B_{1}} G * w(s, x) \partial_{s} v(s, x) d x d s=0,
\end{aligned}
$$

for all $v \in L^{\infty}\left([0, \infty), H^{1}\left(\mathbb{R}^{d}\right)\right)$ with $\partial_{t} v \in L^{\infty}\left([0, \infty), L^{2}\left(\mathbb{R}^{d}\right)\right)$ and $v(t, x)=0$. Setting

$$
v(s, x)=\int_{s}^{t} w(\tau, x) d \tau,
$$

substituting $v$ in (4.12), and using the fact that $\partial_{s} v(s, x)=-w(s, x)$, we obtain

$$
\begin{aligned}
& \int_{0}^{t} \int_{\mathbb{R}^{d}} \Sigma_{1, c}(x) \partial_{s} w(s, x) w(s, x) d x d s-\int_{0}^{t} \int_{\mathbb{R}^{d}} A_{c}(x) \partial_{s} \nabla v(s, x) \nabla v(s, x) d x d s \\
& \quad+\int_{0}^{t} \int_{B_{1} \backslash B_{1 / 2}} \Sigma_{2, c}(x)|w(s, x)|^{2} d x d s+\int_{0}^{t} \int_{B_{2} \backslash B_{1}} G * w(s, x) w(s, x) d x d s=0 .
\end{aligned}
$$

It follows that

$$
\begin{aligned}
& \frac{1}{2} \int_{\mathbb{R}^{d}}\left(\Sigma_{1, c}|w(t, x)|^{2}+A_{c}(x)|\nabla v(0, x)|^{2}\right) d x \\
& \quad+\int_{0}^{t} \int_{B_{1} \backslash B_{1 / 2}} \Sigma_{2, c}(x)|w(s, x)|^{2} d x d s+\int_{0}^{t} \int_{B_{2} \backslash B_{1}} G * w(s, x) w(s, x) d x d s=0,
\end{aligned}
$$

which in particular yields 


$$
\int_{\mathbb{R}^{d}} \Sigma_{1, c}|w(t, x)|^{2} d x=0
$$

or

$$
w(t, x)=0 \quad \text { for a.e. } x \in \mathbb{R}^{d} \text { and all } t \geq 0 .
$$

Here we used the fact that

$$
\int_{0}^{t} \int_{B_{2} \backslash B_{1}} G * w(s, x) w(s, x) d x d s \geq 0
$$

cf. (4.8). This establishes the uniqueness of the weak solution $u$. The proof is complete.

Let $\hat{u}_{c}(k, x)$ be the Fourier transform of $u_{c}$ with respect to time, i.e., ${ }^{7}$

$$
\hat{u}_{c}(k, x):=\frac{1}{\sqrt{2 \pi}} \int_{-\infty}^{\infty} u_{c}(t, x) e^{i k t} d t
$$

We have

Proposition 2. Let $d=2$ or $d=3$, and let $f \in L^{2}\left([0,+\infty) \times \mathbb{R}^{d}\right)$ with compact support. Suppose $u_{c} \in$ $L^{\infty}\left([0,+\infty) ; H^{1}\left(\mathbb{R}^{d}\right)\right)$ with $\partial_{t} u_{c} \in L^{\infty}\left([0,+\infty) ; L^{2}\left(\mathbb{R}^{d}\right)\right)$ is the unique weak solution to $(1.6)$. Then $\hat{u}_{c}(k, \cdot) \in$ $H_{l o c}^{1}\left(\mathbb{R}^{d}\right)$ is the unique outgoing solution to the equation

$$
\operatorname{div}\left(A_{c} \nabla \hat{u}_{c}\right)+k^{2} \Sigma_{c} \hat{u}_{c}=-\hat{f}
$$

for a.e. $k>0$. Moreover,

$$
k \hat{u}_{c}(k, x) \in L_{l o c}^{2}\left([0,+\infty) \times \mathbb{R}^{d}\right) .
$$

We recall that $\hat{u}_{c}$ denotes the Fourier transform of $u_{c}\left(u_{c}\right.$ is extended by 0 for $\left.t<0\right)$.

Outline of Proof. The proof of the first fact is similar to the one of [20, Theorem A1], and is based on the so called limiting absorption principle. A key ingredient, as in [20, (A9)], is the technique from the proof of Proposition 1 where the energy estimate was established. The fact that $k \hat{u}_{c} \in L_{l o c}^{2}\left([0,+\infty) \times \mathbb{R}^{d}\right)$ is obtained as follows. Let $u_{\delta} \in L^{\infty}\left([0,+\infty) ; H^{1}\left(\mathbb{R}^{d}\right)\right)$ with $\partial_{t} u_{\delta} \in L^{\infty}\left([0,+\infty) ; L^{2}\left(\mathbb{R}^{d}\right)\right)$ be the unique weak solution $^{8}$ to

$$
\left\{\begin{array}{cl}
\Sigma_{1, c} \partial_{t t}^{2} u_{\delta}-\operatorname{div}\left(A_{c} \nabla u_{\delta}\right)+\Sigma_{2, c} \partial_{t} u_{\delta}+G * \partial_{t} u_{\delta}+\delta \partial_{t} u_{\delta}=f & \text { in }[0,+\infty) \times \mathbb{R}^{d} \\
\partial_{t} u_{\delta}(t=0)=u_{\delta}(t=0)=0 & \text { in } \mathbb{R}^{d}
\end{array}\right.
$$

Then, as in the proof of Proposition 1,

$$
\delta \int_{0}^{t} \int_{\mathbb{R}^{d}}\left|\partial_{t} u_{\delta}\right|^{2} \leq C
$$

\footnotetext{
7 We extend $u_{c}$ by 0 for $t<0$.

8 The definition of weak solutions for the equation of $u_{\delta}$ is similar to the one for the equation of $u_{c}$.
} 
This implies $\partial_{t} u_{\delta} \in L^{2}\left([0,+\infty) ; L^{2}\left(\mathbb{R}^{d}\right)\right)$, and thus $k \hat{u}_{\delta} \in L^{2}\left([0,+\infty) ; L^{2}\left(\mathbb{R}^{d}\right)\right)=L^{2}\left([0, \infty) \times \mathbb{R}^{d}\right)$. Here $\hat{u}_{\delta}$ denotes the Fourier transform of $u_{\delta}\left(u_{\delta}\right.$ is extended by 0 for $\left.t<0\right)$. As in the proof of [20, Theorem A1], ${ }^{9}$ for almost every $k>0, \hat{u}_{\delta}(k,) \in H^{1}\left(\mathbb{R}^{d}\right)$ is the unique solution to

$$
\operatorname{div}\left(A_{c} \nabla \hat{u}_{\delta}(k, \cdot)\right)+k^{2} \Sigma_{c} \hat{u}_{\delta}(k, \cdot)+i \delta k \hat{u}_{\delta}(k, \cdot)=-\hat{f}(k, \cdot) .
$$

Fix $k_{0}>0$ arbitrary. We have, for $0 \leq k \leq k_{0}$ and for $0<\delta<1$,

$$
\left\|\hat{u}_{\delta}(k, \cdot)\right\|_{L^{2}\left(B_{R}\right)} \leq C k^{-1}\|\hat{f}(k, \cdot)\|_{L^{2}\left(B_{R}\right)}
$$

for some positive constant $C$, independent of $\delta$ and $k$, see Lemma 7 below. Since $f$ has compact support,

$$
\|\hat{f}(k, \cdot)\|_{L^{2}\left(B_{R}\right)} \leq C\|f\|_{L^{2}} .
$$

A combination of (4.15), (4.16), and (4.17) yields

$$
\left(k \hat{u}_{\delta}(k)\right)_{0<\delta<1} \text { is bounded in } L_{l o c}^{2}\left([0,+\infty) \times \mathbb{R}^{d}\right) .
$$

By the limiting absorption principle (see e.g. [11, Section 4.6]) we have, for almost every $k>0$,

$$
\hat{u}_{\delta}(k, \cdot) \rightarrow V(k, \cdot) \text { weakly in } H_{l o c}^{1}\left(\mathbb{R}^{d}\right)
$$

where $V(k, x) \in H_{l o c}^{1}\left(\mathbb{R}^{d}\right)$ is the unique outgoing solution to (4.13). On the other hand (see e.g., the proof of $[20$, Theorem A1] in particular $[20,(\mathrm{~A} 13)])$,

$$
k \hat{u}_{\delta}(k, x) \text { converges to } k V(k, x) \text { in the distributional sense on } \mathbb{R} \times \mathbb{R}^{d} \text {. }
$$

Since $V(k, \cdot)=\hat{u}_{c}(k, \cdot), k>0$, we derive from (4.18), (4.19), and (4.20) that

$$
k \hat{u}_{c} \in L_{l o c}^{2}\left([0,+\infty) \times \mathbb{R}^{d}\right) .
$$

The proof is complete.

In the proof of Proposition 2, we used a simple consequence of the following lemma:

Lemma 7. Given $g \in L^{2}\left(\mathbb{R}^{d}\right)$ with supp $g \subset B_{R_{0}}, 0<\delta<1$, and $0<k<k_{0}$, let $v_{k, \delta} \in H^{1}\left(\mathbb{R}^{d}\right)$ be the unique solution to

$$
\operatorname{div}\left(A_{c} \nabla v_{k, \delta}\right)+\left(k^{2} \Sigma_{c}+i k \delta\right) v_{k, \delta}=g \text { in } \mathbb{R}^{d} .
$$

For any $R_{1}>0$, there exists a positive constant $C_{R_{1}}$ independent of $k, \delta$, and $g$ such that

$$
\left\|v_{k, \delta}\right\|_{L^{2}\left(B_{R_{1}}\right)} \leq C_{R_{1}}\left(|\ln k|^{2}+1\right)\|g\|_{L^{2}} \quad \text { for } d=2
$$

and

$$
\left\|v_{k, \delta}\right\|_{L^{2}\left(B_{R_{1}}\right)} \leq C_{R_{1}}\|g\|_{L^{2}} \quad \text { for } d=3
$$

\footnotetext{
${ }^{9}$ More precisely, [20, (A10) and the following paragraph].
} 
Proof. Under the conditions $k<k_{0}$ and $\delta<1$, the estimates (4.21) and (4.22) follow from a standard contradiction argument if $k$ is bounded below by a positive constant. Lemma 8 below implies these same estimates for sufficiently small $k$, and the proof is complete.

Lemma 8. Let $A \in\left[L^{\infty}\left(\mathbb{R}^{d}\right)\right]^{d \times d}$ and $\Sigma \in L^{\infty}\left(\mathbb{R}^{d}\right), d=2,3$ be such that $A$ is uniformly elliptic, $A=I$ in $\mathbb{R}^{d} \backslash B_{2}, \Sigma=1$ in $\mathbb{R}^{d} \backslash B_{2}, \Re(\Sigma)$ is strictly positive, and $\Im(\Sigma) \geq 0$. Given $g \in L^{2}\left(\mathbb{R}^{d}\right)$ with $\operatorname{supp} g \subset B_{R_{0}}$, and $0<\varepsilon, \delta<1$, let $v_{\varepsilon, \delta} \in H^{1}\left(\mathbb{R}^{d}\right)$ be the unique solution to

$$
\operatorname{div}\left(A \nabla v_{\varepsilon, \delta}\right)+\left(\varepsilon^{2} \Sigma+i \delta\right) v_{\varepsilon, \delta}=g \text { in } \mathbb{R}^{d}
$$

For any $R_{1}>0$, there exist two positive constants $c$ and $C$ independent of $\varepsilon$ and $\delta$ such that if $0<\varepsilon, \delta<c$ then

$$
\left\|v_{\varepsilon, \delta}\right\|_{L^{2}\left(B_{R_{1}}\right)} \leq C|\ln \varepsilon|^{2}\|g\|_{L^{2}} \quad \text { for } d=2
$$

and

$$
\left\|v_{\varepsilon, \delta}\right\|_{L^{2}\left(B_{R_{1}}\right)} \leq C\|g\|_{L^{2}} \quad \text { for } \quad \text { for } d=3
$$

Proof. The proof of this lemma for $d=3$ is simpler than for $d=2$. Essentially the proof for $d=3$ follows along the first third of the argument for $d=2$. For this reason we only present the proof for $d=2$. We may without loss of generality suppose $R_{1}>R_{0}$ (if not, simply increase $R_{1}$ ), and for simplicity of notation we use $R_{0}=4, R_{1}=5$. The proof proceeds by contradiction. Suppose there exist a sequence $\varepsilon_{n} \rightarrow 0$, a sequence $\delta_{n} \rightarrow 0$, and a sequence $\left(g_{n}\right) \subset L^{2}\left(\mathbb{R}^{2}\right)$ such that $\operatorname{supp} g_{n} \subset B_{4}$, and

$$
\lim _{n \rightarrow \infty}\left|\ln \varepsilon_{n}\right|^{2}\left\|g_{n}\right\|_{L^{2}}=0, \quad \text { and } \quad\left\|v_{n}\right\|_{L^{2}\left(B_{5}\right)}=1
$$

Here $v_{n} \in H^{1}\left(\mathbb{R}^{2}\right)$ is the unique solution to

$$
\operatorname{div}\left(A \nabla v_{n}\right)+\left(\varepsilon_{n}^{2} \Sigma+i \delta_{n}\right) v_{n}=g_{n} \text { in } \mathbb{R}^{2} .
$$

Multiplying this equation by $\bar{v}_{n}$ and integrating the obtained expression on $B_{5}$, we have

$$
\int_{B_{5}}\left\langle A \nabla v_{n}, \nabla \bar{v}_{n}\right\rangle-\int_{B_{5}}\left(\varepsilon_{n}^{2} \Sigma+i \delta_{n}\right)\left|v_{n}\right|^{2}=-\int_{B_{5}} g_{n} \bar{v}_{n}+\int_{\partial B_{5}} \frac{\partial v_{n}}{\partial r} \bar{v}_{n} .
$$

Since $\Delta v_{n}+\left(\varepsilon_{n}^{2}+i \delta_{n}\right) v_{n}=0$ in $\mathbb{R}^{2} \backslash B_{4}$ and $v_{n} \in H^{1}\left(\mathbb{R}^{2}\right)$, it follows that ${ }^{10}$

$$
\left\|v_{n}\right\|_{H^{1}\left(B_{R} \backslash B_{9 / 2}\right)} \leq C_{R}\left\|v_{n}\right\|_{H^{1 / 2}\left(\partial B_{9 / 2}\right)} \leq C_{R}\left\|v_{n}\right\|_{L^{2}\left(B_{5} \backslash B_{4}\right)} \leq C_{R} \quad \text { for } R>9 / 2 .
$$

We derive from (4.23) and (4.24) that

$$
\int_{B_{5}}\left|\nabla v_{n}\right|^{2} \leq C
$$

A combination of (4.25) and (4.26) yields

$\overline{10}$ One can use (4.27). below to derive this property. 


$$
\left\|v_{n}\right\|_{H^{1}\left(B_{R}\right)} \leq C_{R} \quad \forall R>0
$$

Thus (after extraction of a subsequence) $v_{n} \rightarrow v$ in $L_{l o c}^{2}\left(\mathbb{R}^{2}\right)$, where $v \in W^{1}\left(\mathbb{R}^{2}\right)$ is a solution to ${ }^{11}$

$$
\operatorname{div}(A \nabla v)=0 \text { in } \mathbb{R}^{2} .
$$

It is clear that $v=\alpha$ for some (complex) constant $\alpha$. For $d=3$ the proof would proceed similarly until this point, where we could automatically conclude that $\alpha$ is zero, and we would have reached a contradiction. In the two dimensional case it requires the following additional argument to show that $\alpha$ is zero. Since $\Delta v_{n}+\hat{\varepsilon}_{n}^{2} v_{n}=0$ in $\mathbb{R}^{2} \backslash B_{4}$ with $\hat{\varepsilon}_{n}^{2}=\varepsilon_{n}^{2}+i \delta_{n}$ and $\Im\left(\hat{\varepsilon}_{n}\right)>0, v_{n} \in H^{1}\left(\mathbb{R}^{2}\right)$ can be represented as

$$
v_{n}(x)=\sum_{l=-\infty}^{\infty} a_{l, n} H_{l}^{(1)}\left(\hat{\varepsilon}_{n}|x|\right) e^{i l \theta} \quad|x|>4,
$$

where $H_{l}^{(1)}$ is the Hankel function of the first kind of order $l$. This implies

$$
v_{n}=v_{0, n}+v_{1, n} \quad|x|>4,
$$

where

$$
v_{0, n}=a_{0, n} H_{0}^{(1)}\left(\hat{\varepsilon}_{n}|x|\right), \quad \text { and } \quad v_{1, n}=\sum_{l \neq 0} a_{l, n} H_{l}^{(1)}\left(\hat{\varepsilon}_{n}|x|\right) e^{i l \theta}, \quad|x|>4
$$

By orthogonality, it is clear that for any $R>4$,

$$
\left\|v_{0, n}\right\|_{H^{1}\left(B_{R} \backslash B_{4}\right)}+\left\|v_{1, n}\right\|_{H^{1}\left(B_{R} \backslash B_{4}\right)} \leq C\left\|v_{n}\right\|_{H^{1}\left(B_{R} \backslash B_{4}\right)} \cdot
$$

After extraction of a subsequence, we may assume that $v_{0, n} \rightarrow \alpha_{0}$ in $L_{l o c}^{2}\left(\mathbb{R}^{2} \backslash B_{4}\right)$ and $v_{1, n} \rightarrow v_{1}$ in $L_{l o c}^{2}\left(\mathbb{R}^{2} \backslash B_{4}\right)$ for some (complex) constant $\alpha_{0}$ and some $v_{1} \in L_{l o c}^{2}\left(\mathbb{R}^{2} \backslash B_{4}\right)$. Therefore,

$$
\alpha=v=\alpha_{0}+v_{1} \quad|x|>4 .
$$

This implies that $v_{1}$ is constant on $\{|x|>4\}$. It follows that $v_{1}=0$ for $|x|>4$ since

$$
\int_{B_{6} \backslash B_{5}} v_{1}=\lim _{n \rightarrow \infty} \int_{B_{6} \backslash B_{5}} v_{1, n}=0 .
$$

As a consequence

$$
\lim _{n \rightarrow \infty} v_{n}=\lim _{n \rightarrow \infty} v_{0, n}=\alpha_{0} \quad \text { in } L_{l o c}^{2}\left(\mathbb{R}^{2} \backslash B_{4}\right)
$$

We have

$$
\int_{\partial B_{R}} \partial_{r} v_{n} \bar{v}_{n}=\int_{B_{R}}\left\langle A \nabla v_{n}, \nabla \bar{v}_{n}\right\rangle-\int_{B_{R}}\left(\varepsilon_{n}^{2} \Sigma+i \delta_{n}\right)\left|v_{n}\right|^{2}+\int_{B_{R}} g_{n} \bar{v}_{n} .
$$

If we let $R \rightarrow \infty$ and consider only the imaginary part, then we obtain

\footnotetext{
11 The proof is similar to the one of Lemma 2.
} 


$$
\Im \int_{\mathbb{R}^{2}}\left(\varepsilon_{n}^{2} \Sigma+i \delta_{n}\right)\left|v_{n}\right|^{2}=\Im \int_{\mathbb{R}^{2}} g_{n} \bar{v}_{n}
$$

Due to the fact that $\hat{\varepsilon}^{n}$ has a positive imaginary part we have that $v_{0, n} \in H^{1}\left(\mathbb{R}^{2}\right)$ (actually it decreases exponentially at $\infty$ ), and so

$$
\int_{\partial B_{5}} \partial_{r} v_{0, n} \bar{v}_{0, n}=-\int_{\mathbb{R}^{2} \backslash B_{5}}\left|\nabla v_{0, n}\right|^{2}+\int_{\mathbb{R}^{2} \backslash B_{5}}\left(\varepsilon_{n}^{2}+i \delta_{n}\right)\left|v_{0, n}\right|^{2},
$$

which leads to

$$
\begin{aligned}
\Im \int_{\partial B_{5}} \partial_{r} v_{0, n} \bar{v}_{0, n} & =\Im \int_{\mathbb{R}^{2} \backslash B_{5}}\left(\varepsilon_{n}^{2}+i \delta_{n}\right)\left|v_{0, n}\right|^{2}=\int_{\mathbb{R}^{2} \backslash B_{5}} \delta_{n}\left|v_{0, n}\right|^{2} \\
& \leq \int_{\mathbb{R}^{2} \backslash B_{5}} \delta_{n}\left|v_{n}\right|^{2} \leq \Im \int_{\mathbb{R}^{2}}\left(\varepsilon_{n}^{2} \Sigma+i \delta_{n}\right)\left|v_{n}\right|^{2} .
\end{aligned}
$$

For the last two inequalities we used the orthogonality of the decomposition (4.28), and the facts that $\Sigma=1$ in $\mathbb{R}^{2} \backslash B_{5}$ and $\Im \Sigma \geq 0$. In combination with (4.23) and (4.30) this gives

$$
\left|\Im \int_{\partial B_{5}} \partial_{r} v_{0, n} \bar{v}_{0, n}\right| \leq\left\|g_{n}\right\|_{L^{2}}\left\|v_{n}\right\|_{L^{2}\left(B_{5}\right)} \leq\left\|g_{n}\right\|_{L^{2}} .
$$

A simple calculation, based on (4.29) and the well-known asymptotics of the Hankel function $H_{0}^{(1)}$ for small argument (see e.g., [1, page 360]), gives

$$
c\left|a_{0, n}\right|^{2} \leq\left|\Im \int_{\partial B_{5}} \partial_{r} v_{0, n} \bar{v}_{0, n}\right|
$$

and so, in combination with (4.31), and (4.23) we get

$$
\left|\ln \varepsilon_{n}\right|^{2}\left|a_{0, n}\right|^{2} \leq C\left|\ln \varepsilon_{n}\right|^{2}\left\|g_{n}\right\|_{L^{2}} \rightarrow 0 \text { as } n \rightarrow \infty \text {. }
$$

This estimate and the formula (4.29) for $v_{0, n}$ now yields

$$
\lim _{n \rightarrow \infty} v_{0, n}=0 \quad \text { on any bounded subset of } \mathbb{R}^{2} \backslash B_{4}
$$

Accordingly we have $\alpha=\alpha_{0}=0$, and so it follows that the $v_{n}$ converge to 0 in $L_{l o c}^{2}\left(\mathbb{R}^{2}\right)$. We have thus reached a contradiction to the fact that $\left\|v_{n}\right\|_{L^{2}\left(B_{5}\right)}=1$, and the proof is complete.

\section{Proof of Theorem 2}

The proof is related to that in [20], however, we shall estimate $\partial_{t} u_{c}-\partial_{t} u$ as a way of getting to $u_{c}-u$. This idea was also used in [19]. Let $\hat{u}_{c}(k, \cdot)$ be the Fourier transform of $u_{c}$ with respect to time. By Proposition 2, for a.e. $k>0, \hat{u}_{c}(k, \cdot) \in H_{l o c}^{1}\left(\mathbb{R}^{d}\right)$ is the unique outgoing solution to

$$
\operatorname{div}\left(A_{c} \nabla \hat{u}_{c}\right)+k^{2} \Sigma_{c} \hat{u}_{c}=-\hat{f}
$$


where $\left(A_{c}, \Sigma_{c}\right)$ is given in (1.4). Moreover,

$$
k \hat{u}_{c} \in L_{l o c}^{2}\left([0,+\infty) \times \mathbb{R}^{d}\right) .
$$

As before we introduce $\tilde{u}_{\varepsilon}(k, x)=\hat{u}_{c}\left(k, F_{\varepsilon}(x)\right)$. Then $\tilde{u}_{\varepsilon} \in H_{l o c}^{1}\left(\mathbb{R}^{d}\right)$ is the unique outgoing solution to

$$
\operatorname{div}\left(A_{\varepsilon} \nabla \tilde{u}_{\varepsilon}\right)+k^{2} \Sigma_{\varepsilon} \tilde{u}_{\varepsilon}=-\hat{f} \text { in } \mathbb{R}^{d} .
$$

Here

$$
A_{\varepsilon}, \Sigma_{\varepsilon}=\left\{\begin{array}{cl}
I, 1 & \text { in } \mathbb{R}^{d} \backslash B_{2}, \\
I, \sigma_{\varepsilon}(x)=1+\sigma_{1, \varepsilon}(x) & \text { in } B_{2} \backslash B_{\varepsilon}, \\
\frac{1}{\varepsilon^{d-2}} I, \frac{1}{\varepsilon^{d}}(1+i / k) & \text { in } B_{\varepsilon} \backslash B_{\varepsilon / 2}, \\
\frac{1}{\varepsilon^{d-2}} a(x / \varepsilon), \frac{1}{\varepsilon^{d}} \sigma(x / \varepsilon) & \text { in } B_{\varepsilon / 2},
\end{array}\right.
$$

and

$$
\sigma_{1, \varepsilon}=\left(F_{\varepsilon}^{-1}\right)_{*} \sigma_{1, c}
$$

Recall that $\sigma_{1, c}$ is given by (1.3) with $\sigma_{D}=\sigma_{N}=1$. Let $\tilde{u}_{1, \varepsilon} \in H_{l o c}^{1}\left(\mathbb{R}^{d}\right)$ be the unique outgoing solution to

$$
\operatorname{div}\left(A_{1, \varepsilon} \nabla \tilde{u}_{1, \varepsilon}\right)+k^{2} \Sigma_{1, \varepsilon} \tilde{u}_{1, \varepsilon}=-\hat{f}
$$

with

$$
A_{1, \varepsilon}, \Sigma_{1, \varepsilon}=\left\{\begin{array}{cl}
I, 1 & \text { in } \mathbb{R}^{d} \backslash B_{\varepsilon}, \\
\frac{1}{\varepsilon^{d-2}} I, \frac{1}{\varepsilon^{d}}(1+i / k) & \text { in } B_{\varepsilon} \backslash B_{\varepsilon / 2}, \\
\frac{1}{\varepsilon^{d-2}} a(x / \varepsilon), \frac{1}{\varepsilon^{d}} \sigma(x / \varepsilon) & \text { in } B_{\varepsilon / 2} .
\end{array}\right.
$$

Finally, let $\hat{u}(k, x)$ be the Fourier transform of $u$ with respect to time; $\hat{u}(k, \cdot) \in H_{l o c}^{1}\left(\mathbb{R}^{d}\right)$ is the unique outgoing solution to

$$
\Delta \hat{u}+k^{2} \hat{u}=-\hat{f} \text { in } \mathbb{R}^{d} .
$$

We first estimate

$$
\int_{0}^{1 / \varepsilon} k\left\|\tilde{u}_{1, \varepsilon}-\hat{u}\right\|_{L^{2}\left(B_{5} \backslash B_{2}\right)} d k .
$$

For this purpose, let $\tilde{U}_{1, \varepsilon}(k, \cdot) \in H_{l o c}^{1}\left(\mathbb{R}^{d}\right)$ be the unique outgoing solution to

$$
\left\{\begin{array}{cl}
\Delta \tilde{U}_{1, \varepsilon}+k^{2} \tilde{U}_{1, \varepsilon}=-\hat{f} & \text { in } \mathbb{R}^{d} \backslash B_{\varepsilon}, \\
\tilde{U}_{1, \varepsilon}=0 & \text { in } B_{\varepsilon},
\end{array}\right.
$$

and define, in all of $\mathbb{R}^{d}$, 


$$
w_{1, \varepsilon}(k, \cdot)=\tilde{U}_{1, \varepsilon}(k, \cdot)-\hat{u}(k, \cdot) \quad \text { and } \quad w_{2, \varepsilon}=\tilde{u}_{1, \varepsilon}(k, \cdot)-\tilde{U}_{1, \varepsilon}(k, \cdot) .
$$

Then $w_{1, \varepsilon}(k, \cdot) \in H_{l o c}^{1}\left(\mathbb{R}^{d}\right)$ is the unique outgoing solution to

$$
\begin{cases}\Delta w_{1, \varepsilon}+k^{2} w_{1, \varepsilon}=0 & \text { in } \mathbb{R}^{d} \backslash B_{\varepsilon} \\ w_{1, \varepsilon}=-\hat{u} & \text { in } B_{\varepsilon}\end{cases}
$$

and $w_{2, \varepsilon} \in H_{l o c}^{1}\left(\mathbb{R}^{d}\right)$ is the unique outgoing solution to

$$
\begin{cases}\Delta w_{2, \varepsilon}+k^{2} w_{2, \varepsilon}=0 & \text { in } \mathbb{R}^{d} \backslash B_{\varepsilon} \\ \nabla \cdot\left(A_{1, \varepsilon} \nabla w_{2, \varepsilon}\right)+k^{2} \Sigma_{1, \varepsilon} w_{2, \varepsilon}=0 & \text { in } B_{\varepsilon} \\ \left.\frac{\partial w_{2, \varepsilon}}{\partial r}\right|_{\text {ext }}-\left.\frac{1}{\varepsilon^{d-2}} \frac{\partial w_{2, \varepsilon}}{\partial r}\right|_{\text {int }}=-\frac{\partial \tilde{U}_{1, \varepsilon}}{\partial r} & \text { on } \partial B_{\varepsilon} .\end{cases}
$$

We first estimate $w_{1, \varepsilon}$. By Lemma 1 and the theory of regularity of elliptic equations, we have, for $d=3$ or $(d=2$ and $k>1 / 2)$

$$
\frac{1}{k+1}\left\|\nabla^{2} \hat{u}(k, \cdot)\right\|_{L^{2}\left(B_{2}\right)}+\|\nabla \hat{u}(k, \cdot)\|_{L^{2}\left(B_{2}\right)}+(k+1)\|\hat{u}(k, \cdot)\|_{L^{2}\left(B_{2}\right)} \leq C\|\hat{f}(k, \cdot)\|_{L^{2}}
$$

and for $(0<k<1 / 2$ and $d=2)$

$$
\left\|\nabla^{2} \hat{u}(k, \cdot)\right\|_{L^{2}\left(B_{2}\right)}+\|\nabla \hat{u}(k, \cdot)\|_{L^{2}\left(B_{2}\right)}+\|\hat{u}(k, \cdot)\|_{L^{2}\left(B_{2}\right)} \leq C|\ln k|\|\hat{f}(k, \cdot)\|_{L^{2}}
$$

Here and in the remainder of this proof, $C$ denotes a positive constant independent of $\varepsilon, k$, and $f$. Since $\Delta \hat{u}(k, \varepsilon \cdot)+k^{2} \varepsilon^{2} \hat{u}(k, \varepsilon \cdot)=0$ in $B_{2}$, it follows that

$$
\int_{B_{1}}|\nabla \hat{u}(k, \varepsilon \cdot)|^{2} \leq C \max \left\{1, \varepsilon^{2} k^{2}\right\} \int_{B_{2}}|\hat{u}(k, \varepsilon \cdot)|^{2} \leq C\|\hat{u}(k, \cdot)\|_{L^{\infty}\left(B_{2}\right)}^{2},
$$

for $0<k<1 / \varepsilon$. Using (5.7) and (5.8), we derive that for $0<k<1 / \varepsilon$,

$$
\|\hat{u}(k, \varepsilon \cdot)\|_{H^{1 / 2}\left(\partial B_{1}\right)} \leq C(k+1)\|\hat{f}(k, \cdot)\|_{L^{2}} \text { for } d=3,
$$

and

$$
\|\hat{u}(k, \varepsilon \cdot)\|_{H^{1 / 2}\left(\partial B_{1}\right)} \leq C(k+1) \varphi(k)\|\hat{f}(k, \cdot)\|_{L^{2}} \text { for } d=2 .
$$

Here

$$
\varphi(k)=1 \text { if } k>1 / 2, \quad \text { and } \quad \varphi(k)=|\ln k| \text { if } 0<k<1 / 2 .
$$

Applying Lemma 2 and rescaling, we have for $0<k<1 / \varepsilon$,

$$
\left\|w_{1, \varepsilon}(k, \cdot)\right\|_{L^{2}\left(B_{5} \backslash B_{2}\right)} \leq C \varepsilon(k+1)\|\hat{f}(k, \cdot)\|_{L^{2}} \text { for } d=3,
$$

and

$$
\left\|w_{1, \varepsilon}(k, \cdot)\right\|_{L^{2}\left(B_{5} \backslash B_{2}\right)} \leq C \frac{\left|H_{0}^{(1)}(k)\right|}{\left|H_{0}^{(1)}(k \varepsilon)\right|}(k+1) \varphi(k)\|\hat{f}(k, \cdot)\|_{L^{2}} \text { for } d=2 .
$$


Since

$$
\frac{\left|H_{0}^{(1)}(k)\right|}{\left|H_{0}^{(1)}(k \varepsilon)\right|} \leq C \frac{\min \left\{k^{-1 / 2},|\ln k|+2\right\}}{|\ln (k \varepsilon)|+2} \text { for } 0<k \varepsilon<1
$$

and thus

$$
\frac{\left|H_{0}^{(1)}(k)\right|}{\left|H_{0}^{(1)}(k \varepsilon)\right|} \leq C \begin{cases}\frac{|\ln k|+2}{|\ln \varepsilon|+2}, & k \leq 1, \\ \frac{1}{|\ln \varepsilon|+2}, & 1 \leq k \leq 1 / \varepsilon\end{cases}
$$

we have

$$
k \frac{\left|H_{0}^{(1)}(k)\right|}{\left|H_{0}^{(1)}(k \varepsilon)\right|} \varphi(k) \leq C \frac{k\left(|\ln k|^{2}+1\right)}{|\ln \varepsilon|+2},
$$

for $0<k<1 / \varepsilon$. It now follows from (5.11) and (5.12) that, in the range $0<k<1 / \varepsilon$,

$$
k\left\|w_{1, \varepsilon}(k, \cdot)\right\|_{L^{2}\left(B_{5} \backslash B_{2}\right)} \leq C \varepsilon k(k+1)\|\hat{f}(k, \cdot)\|_{L^{2}} \text { for } d=3,
$$

and

$$
k\left\|w_{1, \varepsilon}(k, \cdot)\right\|_{L^{2}\left(B_{5} \backslash B_{2}\right)} \leq \frac{C}{|\ln \varepsilon|} k(k+1)\left(|\ln k|^{2}+1\right)\|\hat{f}(k, \cdot)\|_{L^{2}} \text { for } d=2 .
$$

We next estimate $w_{2, \varepsilon}$. Applying Lemma 3, we have, for $0<k<1 / \varepsilon$,

$$
\left\|w_{2, \varepsilon}(k, \varepsilon \cdot)\right\|_{L^{2}\left(B_{5} \backslash B_{1}\right)} \leq C \max \{1, \varepsilon / k\}\left\|\frac{\partial}{\partial r}\left(\tilde{U}_{1, \varepsilon}(k, \varepsilon \cdot)\right)\right\|_{H^{-1 / 2}\left(\partial B_{1}\right)} \text { for } d=3
$$

and

$$
\left\|w_{2, \varepsilon}(k, \varepsilon \cdot)\right\|_{L^{2}\left(B_{5} \backslash B_{1}\right)} \leq C \max \{k, 1 / k\}\left\|\frac{\partial}{\partial r}\left(\tilde{U}_{1, \varepsilon}(k, \varepsilon \cdot)\right)\right\|_{H^{-1 / 2}\left(\partial B_{1}\right)} \text { for } d=2 .
$$

For $0<\varepsilon k<1$, the standard trace estimate, and a classical interior elliptic estimate, yield

$$
\left\|w_{2, \varepsilon}(k, \varepsilon \cdot)\right\|_{H^{1 / 2}\left(\partial B_{2}\right)} \leq C\left\|w_{2, \varepsilon}(k, \varepsilon \cdot)\right\|_{H^{1}\left(B_{4} \backslash B_{3 / 2}\right)} \leq C\left\|w_{2, \varepsilon}(k, \varepsilon \cdot)\right\|_{L^{2}\left(B_{5} \backslash B_{1}\right)}
$$

and so by use of Lemma 2, (5.16), (5.17) and a scaling argument, it follows that

$$
\begin{aligned}
\left\|w_{2, \varepsilon}(k, \cdot)\right\|_{L^{2}\left(B_{5} \backslash B_{2}\right)} & =\varepsilon^{3 / 2}\left\|w_{2, \varepsilon}(k, \varepsilon \cdot)\right\|_{L^{2}\left(B_{5 / \varepsilon} \backslash B_{2 / \varepsilon}\right)} \\
& \leq C \varepsilon\left\|w_{2, \varepsilon}(k, \varepsilon \cdot)\right\|_{L^{2}\left(B_{5} \backslash B_{1}\right)} \\
& \leq C \varepsilon \max \{1, \varepsilon / k\}\left\|\frac{\partial}{\partial r}\left(\tilde{U}_{1, \varepsilon}(k, \varepsilon \cdot)\right)\right\|_{H^{-1 / 2}\left(\partial B_{1}\right)} \text { for } d=3,
\end{aligned}
$$

and 


$$
\begin{aligned}
\left\|w_{2, \varepsilon}(k, \cdot)\right\|_{L^{2}\left(B_{5} \backslash B_{2}\right)} & =\varepsilon\left\|w_{2, \varepsilon}(k, \varepsilon \cdot)\right\|_{L^{2}\left(B_{5 / \varepsilon} \backslash B_{2 / \varepsilon}\right)} \\
& \leq C \frac{\left|H_{0}^{(1)}(k)\right|}{\left|H_{0}^{(1)}(\varepsilon k)\right|}\left\|w_{2, \varepsilon}(k, \varepsilon \cdot)\right\|_{L^{2}\left(B_{5} \backslash B_{1}\right)} \\
& \leq C \frac{\left|H_{0}^{(1)}(k)\right|}{\left|H_{0}^{(1)}(\varepsilon k)\right|} \max \{k, 1 / k\}\left\|\frac{\partial}{\partial r}\left(\tilde{U}_{1, \varepsilon}(k, \varepsilon \cdot)\right)\right\|_{H^{-1 / 2}\left(\partial B_{1}\right)} \text { for } d=2 .
\end{aligned}
$$

We have

$$
\left\|\frac{\partial}{\partial r}\left(\tilde{U}_{1, \varepsilon}(k, \varepsilon \cdot)\right)\right\|_{H^{-1 / 2}\left(\partial B_{1}\right)} \leq\left\|\frac{\partial}{\partial r}\left(w_{1, \varepsilon}(k, \varepsilon \cdot)\right)\right\|_{H^{-1 / 2}\left(\partial B_{1}\right)}+\left\|\frac{\partial}{\partial r}(\hat{u}(k, \varepsilon \cdot))\right\|_{H^{-1 / 2}\left(\partial B_{1}\right)} .
$$

Applying Lemma 2 to $w_{1, \varepsilon}(k, \varepsilon \cdot)$ and using (5.9) and (5.10), we obtain, for $0<k<1 / \varepsilon$,

$$
\left\|\frac{\partial}{\partial r}\left(\tilde{U}_{1, \varepsilon}(\varepsilon \cdot)\right)\right\|_{H^{-1 / 2}\left(\partial B_{1}\right)} \leq C(k+1)\|\hat{f}(k, \cdot)\|_{L^{2}} \text { for } d=3,
$$

and

$$
\left\|\frac{\partial}{\partial r}\left(\tilde{U}_{1, \varepsilon}(\varepsilon \cdot)\right)\right\|_{H^{-1 / 2}\left(\partial B_{1}\right)} \leq C(k+1) \varphi(k)\|\hat{f}(k, \cdot)\|_{L^{2}} \text { for } d=2
$$

It now follows from (5.18) and (5.19) that, for $0<k<1 / \varepsilon$,

$$
k\left\|w_{2, \varepsilon}\right\|_{L^{2}\left(B_{5} \backslash B_{2}\right)} \leq C \varepsilon(k+1) \max \{k, \varepsilon\}\|\hat{f}(k, \cdot)\|_{L^{2}} \text { for } d=3
$$

and

$$
k\left\|w_{2, \varepsilon}\right\|_{L^{2}\left(B_{5} \backslash B_{2}\right)} \leq \frac{C(k+1)}{|\ln \varepsilon|}\left(|\ln k|^{2}+1\right) \max \left\{1, k^{2}\right\}\|\hat{f}(k, \cdot)\|_{L^{2}} \text { for } d=2 .
$$

For the last estimate we also used (5.13). A combination of (5.14), (5.15), (5.20), and (5.21) yields

$$
\int_{0}^{1 / \varepsilon} k\left\|\tilde{u}_{1, \varepsilon}-\hat{u}\right\|_{L^{2}\left(B_{5} \backslash B_{2}\right)} d k \leq C \varepsilon \int_{0}^{1 / \varepsilon}(k+1)^{2}\|\hat{f}(k, \cdot)\|_{L^{2}} \leq C \varepsilon\|f\| \quad \text { if } d=3,
$$

and

$$
\begin{aligned}
\int_{0}^{1 / \varepsilon} k\left\|\tilde{u}_{1, \varepsilon}-\hat{u}\right\|_{L^{2}\left(B_{5} \backslash B_{2}\right)} d k & \leq \frac{C}{|\ln \varepsilon|} \int_{0}^{1 / \varepsilon}\left(|\ln k|^{2}+1\right)(1+k)^{3}\|\hat{f}(k, \cdot)\|_{L^{2}} \\
& \leq \frac{C}{|\ln \varepsilon|}\|f\| \quad \text { if } d=2,
\end{aligned}
$$

where $\|f\|$ is the norm introduced in the statement of Theorem 2.

We next estimate $\left\|\tilde{u}_{\varepsilon}(k, \cdot)-\tilde{u}_{1, \varepsilon}(k, \cdot)\right\|_{L^{2}\left(B_{5} \backslash B_{2}\right)}$ for $k$ of order up to $1 / \varepsilon$. We already know that $\Im\left(\sigma_{1, \varepsilon}\right)>0$ for $k>0$, and from Lemma 4 and the fact that $k_{\varepsilon}>c_{*} / \varepsilon^{d / 2}$ we have

$$
\left|\sigma_{1, \varepsilon}\right| \leq \frac{C}{\varepsilon^{d-1} k_{\varepsilon}^{2}} \leq C_{0} \varepsilon
$$


for $0<k<\frac{c_{*}}{2} \varepsilon^{-1}$. Applying Lemma 5 and the first part of Lemma 6 to $\tilde{u}_{\varepsilon}-\tilde{u}_{1, \varepsilon}\left(\right.$ with $\left.g=-k^{2} \sigma_{1, \varepsilon} \tilde{u}_{1, \varepsilon}\right)$ we obtain

$$
k\left\|\tilde{u}_{\varepsilon}(k, \cdot)-\tilde{u}_{1, \varepsilon}(k, \cdot)\right\|_{L^{2}\left(B_{5} \backslash B_{2}\right)} \leq C\left(k^{2}+1\right) k^{2} \sup \left|\sigma_{1, \varepsilon}\right|\left\|\tilde{u}_{1, \varepsilon}(k, \cdot)\right\|_{L^{2}\left(B_{2} \backslash B_{\varepsilon}\right)}
$$

for $0<k<\frac{\lambda}{C_{0} \varepsilon}(\lambda$ is the constant from Lemma 6). A combination of (5.24) and (5.25) yields

$$
k\left\|\tilde{u}_{\varepsilon}(k, \cdot)-\tilde{u}_{1, \varepsilon}(k, \cdot)\right\|_{L^{2}\left(B_{5} \backslash B_{2}\right)} \leq C k^{2} \varepsilon\left(k^{2}+1\right)\left\|\tilde{u}_{1, \varepsilon}(k, \cdot)\right\|_{L^{2}\left(B_{2} \backslash B_{\varepsilon}\right)},
$$

for $0<k<\lambda_{0} / \varepsilon$, with $\lambda_{0}=\min \left\{1, c_{*} / 2, \lambda / C_{0}\right\}$. Similarly, applying Lemma 5 and the first part of Lemma 6 to the function $\hat{u}_{1, \varepsilon}$ (with $g=-f$ and coefficients $A_{1, \varepsilon}, \Sigma_{1, \varepsilon}$, i.e., $A_{\varepsilon}$, $\Sigma_{\varepsilon}$ with $\sigma_{1, \varepsilon}=0$ ) we obtain

$$
k\left\|\tilde{u}_{1, \varepsilon}(k, \cdot)\right\|_{L^{2}\left(B_{5} \backslash B_{2}\right)} \leq C\left(k^{2}+1\right)\|\hat{f}(k, \cdot)\|_{L^{2}},
$$

for $0<k<\lambda_{0} / \varepsilon$. A combination of (5.26) and (5.27) yields

$$
\int_{0}^{\lambda_{0} / \varepsilon} k\left\|\tilde{u}_{\varepsilon}-\tilde{u}_{1, \varepsilon}\right\|_{L^{2}\left(B_{5} \backslash B_{2}\right)} d k \leq C \varepsilon \int_{0}^{\lambda_{0} / \varepsilon}(k+1)^{5}\|\hat{f}(k, \cdot)\|_{L^{2}} \leq C \varepsilon\|f\| .
$$

We now consider the regime $k>\lambda_{0} / \varepsilon$. From the second part of Lemma 6 , and the remark following, we have

$$
k\left\|\tilde{u}_{\varepsilon}(k, \cdot)\right\|_{L^{2}\left(B_{5} \backslash B_{2}\right)} \leq C\left(k^{2}+\frac{k \chi_{1}^{2}}{\chi_{2}}\right)\|\hat{f}(k, \cdot)\|_{L^{2}} \leq \frac{C}{\lambda_{0}} \varepsilon\left(k^{3}+\frac{k^{2} \chi_{1}^{2}}{\chi_{2}}\right)\|\hat{f}(k, \cdot)\|_{L^{2}} .
$$

On the other hand, using Lemma 1 we have

$$
k\|\hat{u}(k, \cdot)\|_{L^{2}\left(B_{5} \backslash B_{2}\right)} \leq C\|\hat{f}(k, \cdot)\|_{L^{2}} \leq \frac{C}{\lambda_{0}} \varepsilon k\|\hat{f}(k, \cdot)\|_{L^{2}},
$$

for $k>\lambda_{0} / \varepsilon$. Lemma 4 yields

$$
\frac{k^{2} \chi_{1}^{2}}{\chi_{2}} \leq C k^{2} \frac{1}{\varepsilon^{2(d-1)} k^{2}} \frac{\max \left\{k_{\varepsilon}^{4}, k^{4}\right\}}{k} \leq C \frac{\max \left\{k_{\varepsilon}^{4}, k^{4}\right\}}{k \varepsilon^{2(d-1)}} \leq C\left(k^{2 d+1}+k^{2 d-3} k_{\varepsilon}^{4}\right)
$$

for $k>\lambda_{0} / \varepsilon$. We derive from (5.29), (5.30), and (5.31) that

$$
\int_{\lambda_{0} / \varepsilon}^{\infty} k\left\|\tilde{u}_{\varepsilon}-\hat{u}\right\|_{L^{2}\left(B_{5} \backslash B_{2}\right)} d k \leq C \varepsilon \int_{\lambda_{0} / \varepsilon}^{\infty}\left(k^{2 d+1}+k^{2 d-3} k_{\varepsilon}^{4}\right)\|\hat{f}(k, \cdot)\|_{L^{2}}
$$

or

$$
\int_{\lambda_{0} / \varepsilon}^{\infty} k\left\|\tilde{u}_{\varepsilon}-\hat{u}\right\|_{L^{2}} d k \leq C \varepsilon\|f\|
$$

A combination of (5.22), (5.23), (5.28), and (5.32) now gives

$$
\int_{0}^{\infty} k\left\|\tilde{u}_{\varepsilon}-\hat{u}\right\|_{L^{2}\left(B_{5} \backslash B_{2}\right)} d k \leq C \varepsilon\|f\| \quad \text { if } d=3
$$


and

$$
\int_{0}^{\infty} k\left\|\tilde{u}_{\varepsilon}-\hat{u}\right\|_{L^{2}\left(B_{5} \backslash B_{2}\right)} d k \leq \frac{C}{|\ln \varepsilon|}\|f\| \quad \text { if } d=2 .
$$

Therefore, since $\tilde{u}_{\varepsilon}(k, \cdot)=\hat{u}_{c}(k, \cdot)$ outside $B_{2}\left(\right.$ and since $u_{c}$ and $u$ are real, so that $\hat{u}_{c}(-k, \cdot)-\hat{u}(-k, \cdot)=$ $\left.\overline{\hat{u}_{c}(k, \cdot)-\hat{u}(k, \cdot)}\right)$ it follows that

$$
\sup _{t>0}\left\|\partial_{t} u_{c}(t, \cdot)-\partial_{t} u(t, \cdot)\right\|_{L^{2}\left(B_{5} \backslash B_{2}\right)} \leq C \varepsilon\|f\| \quad \text { if } d=3
$$

and

$$
\sup _{t>0}\left\|\partial_{t} u_{c}(t, \cdot)-\partial_{t} u(t, \cdot)\right\|_{L^{2}\left(B_{5} \backslash B_{2}\right)} \leq \frac{C}{|\ln \varepsilon|}\|f\| \quad \text { if } d=2 .
$$

From this we conclude

$$
\sup _{0<t<T}\left\|u_{c}-u\right\|_{L^{2}\left(B_{5} \backslash B_{2}\right)} \leq C T \varepsilon\|f\| \quad \text { if } d=3
$$

and

$$
\sup _{0<t<T}\left\|u_{c}-u\right\|_{L^{2}\left(B_{5} \backslash B_{2}\right)} \leq \frac{C T}{|\ln \varepsilon|}\|f\| \quad \text { if } d=2
$$

The proof of Theorem 2 is complete.

\section{Acknowledgement}

The authors would like to thank Michael Weinstein for introducing them to the Drude-Lorentz model, and for related discussions. The work of H-M. Nguyen was partially supported by NSF grant DMS-1201370 and by the Alfred P. Sloan Foundation. The work of M.S. Vogelius was partially supported by NSF grant DMS-1211330, and by the NSF IR/D program while serving at the National Science Foundation.

\section{References}

[1] M. Abramowitz, I. Stegun, Handbook of Mathematical Functions, National Bureau of Standards Applied Mathematics Series, vol. 55, 1964.

[2] T.A. Burton, Volterra Integral and Differential Equations, Mathematics in Science and Engineering, vol. 167, Academic Press, Inc., Orlando, FL, 1983.

[3] W. Cai, U.K. Chettiar, A.V. Kildishev, V.M. Shalaev, Optical cloaking with metamaterials, Nat. Photonics 1 (2007) 224-227.

[4] A. Greenleaf, Y. Kurylev, M. Lassas, G. Uhlmann, Improvement of cylindrical cloaking with the SHS lining, Opt. Express 15 (2007) 12717.

[5] A. Greenleaf, Y. Kurylev, M. Lassas, G. Uhlmann, Electromagnetic wormholes via handlebody constructions, Commun. Math. Phys. 281 (2008) 369-385.

[6] A. Greenleaf, M. Lassas, G. Uhlmann, On nonuniqueness for Calderon's inverse problem, Math. Res. Lett. 10 (2003) 685-693.

[7] H. Haddar, P. Joly, H-M. Nguyen, Generalized impedance boundary conditions for scattering by strongly absorbing obstacles: the scalar case, Math. Models Methods Appl. Sci. 15 (2005) 1273-1300.

[8] J.D. Jackson, Classical Electrodynamics, third ed., John Willey, 2001.

[9] R.V. Kohn, D. Onofrei, M.S. Vogelius, M.I. Weinstein, Cloaking via change of variables for the Helmholtz equation, Commun. Pure Appl. Math. 63 (2010) 973-1016.

[10] R.V. Kohn, H. Shen, M.S. Vogelius, M.I. Weinstein, Cloaking via change of variables in electric impedance tomography, Inverse Probl. 24 (2008) 015016. 
[11] R. Leis, Initial-Boundary Value Problems in Mathematical Physics, B. G. Teubner/John Wiley-Sons, Ltd., Stuttgart/ Chichester, 1986.

[12] U. Leonhardt, Optical conformal mapping, Science 312 (2006) 1777-1780.

[13] U. Leonhardt, T. Tyc, Broadband invisibility by non-Euclidean cloaking, Science 323 (2009) 110-112.

[14] J. Li, H. Liu, L. Rondi, G. Uhlmann, Regularized transformation-optics cloaking for Helmholtz equation: from partial cloak to full cloak, Commun. Math. Phys. 335 (2015) 671-712.

[15] C.S. Morawetz, D. Ludwig, An inequality for the reduced wave operator and the justification of geometrical optics, Commun. Pure Appl. Math. 21 (1968) 187-203.

[16] H-M. Nguyen, Cloaking via change of variables for the Helmholtz equation in the whole space, Commun. Pure Appl. Math. 63 (2010) 1505-1524.

[17] H-M. Nguyen, Approximate cloaking for the Helmholtz equation via transformation optics and consequences for perfect cloaking, Commun. Pure Appl. Math. 65 (2012) 155-186.

[18] H-M. Nguyen, On a regularized scheme for approximate acoustic cloaking using transformation optics, SIAM J. Math. Anal. 45 (2013) 3034-3049.

[19] H-M. Nguyen, L. Nguyen, Generalized impedance boundary conditions for scattering by strongly absorbing obstacles for the full wave equation: the scalar case, Math. Models Methods Appl. Sci. 25 (2015) 1927-1960.

[20] H-M. Nguyen, M.S. Vogelius, Approximate cloaking for the full wave equation via change of variables, SIAM J. Math. Anal. 44 (2012) 1894-1924.

[21] H-M. Nguyen, M.S. Vogelius, Full range scattering estimates and their application to cloaking, Arch. Ration. Mech. Anal. 203 (2012) 769-807.

[22] L.E. Payne, H.F. Weinberger, New bounds for solutions of second order elliptic partial differential equations, Pac. J. Math. 8 (1958) 551-573.

[23] J.B. Pendry, D. Schurig, D.R. Smith, Controlling electromagnetic fields, Science 312 (2006) 1780-1782.

[24] B. Perthame, L. Vega, Morrey-Campanato estimates for Helmholtz equations, J. Funct. Anal. 164 (1999) $340-355$.

[25] F. Rellich, Darstellung der Eigenwerte von $\Delta u+\lambda u=0$ durch ein Randintegral, Math. Z. 46 (1940) 635-636.

[26] Z. Ruan, M. Yan, C.M. Neff, M. Qiu, Ideal cylindrical cloak: perfect but sensitive to tiny perturbations, Phys. Rev. Lett. 99 (2007) 113903.

[27] D. Schurig, J.J. Mock, J. Justice, S.A. Cummer, J.B. Pendry, A.F. Starr, D.R. Smith, Metamaterial electromagnetic cloak at microwave frequencies, Science 314 (2006) 1133628.

[28] J.S. Toll, Causality and the dispersion relation: logical foundations, Phys. Rev. 104 (1956) 1760-1770. 